\title{
Chapter 26 \\ Liquid Micro Jet Studies of the Vacuum Surface of Water and of Chemical Solutions by Molecular Beams and by Soft X-Ray Photoelectron Spectroscopy
}

\author{
Manfred Faubel
}

Liquid water, with a vapor pressure of 6.1 mbar at freezing point, is rapidly evaporating in high vacuum, rapidly cooling off by the evaporational cooling, and is freezing to ice almost instantly.

Historically, this was noted already in the very earliest experiments with vacuum by Otto Guericke, the inventor of a first vacuum pump, and by Robert Boyle. In a contemporary, serendipitous report, published in 1664 in Nurnberg, G. Scotus in his "Technica curiosa" [1] is listing a number of noteworthy, miraculous and curious observations within the newly created vacuum space inside a bell jar, like: light is penetrating vacuum, the sound of a ringing bell can not penetrate to the outside, a candle flame is extinguishing, but, gun powder can be ignited inside a vacuum; small animals such as a mouse are dying quickly in a evacuated bell jar vacuum while certain insects can survive for a while. And eventually, as an item in his volume 2, Chap. 15, "Experimentum XXXVIII. Aqua intra evacuatum Recipientum congelatur", (experiment no. 38. Water inside the evacuated recipient is freezing to ice). With scholar diligence G. Scotus has annotated that with the "new" mechanically improved vacuum pump of Robert Boyle the water is freezing in vacuum while it was not observed to freeze in the equipment provided by Otto Guericke to the laboratory of G. Scotus. From our modern view we can conclude that Boyle's pump did reach a vacuum better than 6.1 mbar, the vapor pressure of ice at freezing, while Guericke's original pump, known to have been operated with water lubricated seals and not yet with fat or oil, did stop pumping slightly above the freezing point vapor pressure of water.

Thus, for centuries to come-including the first 80 years of modern age "Otto Stern type experiments"-liquid aqueous solutions were not considered as suitable

M. Faubel (凶)

Max-Planck-Institut für Dynamik und Selbstorganisation, Göttingen, Germany

e-mail: manfred.faubel@ds.mpg.de; Manfred.Faubel@mpibpc.mpg.de

B. Friedrich and H. Schmidt-Böcking (eds.), Molecular Beams in Physics and Chemistry, https://doi.org/10.1007/978-3-030-63963-1_26 
for research employing vacuum or any molecular beams technology or ultrahigh vacuum surface diagnostics.

\section{The Free Vacuum Surface of Water Microjets}

With this unfavorable veredictum for water in vacuum in mind, for advancing experiments successfully it is helpful to have a very close look at the dynamical processes on the surface of liquid water [2-4]. Here three principal problems dominate: (1) due to the high vapor pressure and the rapid evaporation a liquid water surface is always overcast by a dense gas cloud associated with the steady vapor stream into the adjacent vacuum space. At the minimum pressure of 6.1 mbar for liquid water, just above freezing, the molecular mean free path in this vapor is only $l=10 \mu \mathrm{m}$. At a first glance this is preventing molecular beam type experiments at the surface, unless the geometrical extension of a water surface experiment could be shrunk to total dimensions smaller than $10 \mu \mathrm{m}$. Furthermore, (2) the un-obstructed unilateral free flow of molecules leaving the liquid surface with a Maxwellian velocity distribution at an average velocity of water molecules of approximately $1000 \mathrm{~m} / \mathrm{s}$ is resulting in a massive gas flux of $600 \mathrm{mbar} \mathrm{L} / \mathrm{s} / \mathrm{cm}^{2}$ for each square-centimeter of liquid surface, requiring extremely large pumping capacities in order to sustain a moderately decent vacuum. This vapor flow density, equivalent to $0.027 \mathrm{Mol} /\left(\mathrm{s} \mathrm{cm}^{2}\right)$, that is $0.48 \mathrm{~g} \mathrm{~s}^{-1}$ $\mathrm{cm}^{-2} \mathrm{H}_{2} \mathrm{O}$, corresponds on the liquid side of the separating surface to a liquid water surface ablation rate of $4.8 \mathrm{~mm} / \mathrm{s}$. (3) Considering the water heat of vaporization of $40 \mathrm{~kJ} / \mathrm{mol}$ the surface cooling rate by evaporation is of the order of $1 \mathrm{~kW} / \mathrm{cm}^{2}$ for liquid water in free vacuum, inducing a very rapid freezing to ice of the vacuum exposed liquid surface.

In a practicable, efficient way these three challenges can be resolved with the use of a fast flowing very thin cylindrical liquid jet in high vacuum, a method developed since the 1980's (Faubel, Schlemmer, Toennies) and, gratefully, first published in a Zeitschrift für Physik "Otto Stern Centenial Issue”, 1988 [2].

For a thin liquid jet of water with diameter $\mathrm{D}$ smaller than the vapor mean free path $\mathrm{l}_{\mathrm{H}_{2} \mathrm{O}}=10 \mu \mathrm{m}$ : (1) Knudsen conditions, D/1 $\mathrm{H}_{\mathrm{H}_{2} \mathrm{O}}<0.5$, are met for collision-free "high vacuum" gas flow; (2) the total liquid surface is small and, thus, the vapor flow into the vacuum system is restricted to values lower than $0.5 \mathrm{mbar} \mathrm{L} / \mathrm{s}$, while (3) the rapid outflow of liquid from the thermostat controlled nozzle is continuously replacing the evaporation-cooled water surface before it can freeze to ice. One further benefit of the microjet technique is in providing an ultra-clean liquid surface due to the rapid replacement of the vacuum exposed surface section. A $1 \mathrm{~mm}$ long microjet surface section, flowing with a speed of $100 \mathrm{~m} / \mathrm{s}$, is replaced within $10 \mu \mathrm{sec}$. Usually, in surface science, for the handling of solid state surfaces, technically demanding ultrahigh vacuum conditions with pressures lower than $\mathrm{p}_{\mathrm{Vac}}=10^{-10} \mathrm{mbar}$ are required to prevent surface coverage by impacting, and sticking, gas molecules during a typical experimental observation session of $\mathrm{t}_{\text {expos }} \geq 1000 \mathrm{~s}$, according to Langmuir's surface coverage relation of: $\mathrm{p}_{\mathrm{Vac}} \cdot \mathrm{t}_{\mathrm{expos}}=10^{-6} \mathrm{mbar} \mathrm{s}$ for coverage with 1 monolayer. On 
fast microjets, however, with short lived, continuously replacing surface the exposure time scales are $\mathrm{t}_{\text {expos }}{ }^{\prime}=10 \mu \mathrm{s}$, only. Therefore, a background gas pressure of $\mathrm{p}_{\mathrm{Vac}}$ ' $=10^{-3}$ mbar, with $\mathrm{p}_{\mathrm{Vac}}{ }^{\prime} \cdot \mathrm{t}_{\text {expos }}{ }^{\prime}=10^{-8}$ mbar $\mathrm{s}$ implying $1 \%$ surface coverage, is already the equivalent to perfectly clean, "ultrahigh vacuum" conditions from a point of view of surface coverage times.

In Fig. 1 the vacuum microjet setup scheme is depicted, showing a high-speed photography of a real jet, at $10 \mathrm{~ns}$ exposure time, and a sketched-in stream-tube along a vapor expansion line. A representative ensemble of gas molecules moving outward on a streamline progressively is increasing its available volume (blunt cone shape enclosures), indicated here for two different time instances, in order to illustrate the decrease in local gas density $n(r) \sim n_{0}\left(r_{0} / r\right)^{2}$ in proportion to the quadratic radial distance $r$ from the microjet. This results in a rapid increase in the local molecular mean free path $1=1 /\left(\mathrm{n}(\mathrm{r}) \cdot \sigma_{\text {collision }}\right)$ in proportion to $\mathrm{r}^{2}$. At the liquid surface position, at $\mathrm{r}_{0}=\mathrm{D} / 2$ given by the microjet diameter, the equilibrium vapor pressure density $\mathrm{n}_{0}$ is assumed. Relevant for describing vacuum conditions (M. Knudsen 1905) is the number of molecular collisions on their path through a vacuum space: $\mathrm{dN}=$ $\mathrm{dr} / \mathrm{l}_{\mathrm{H}_{2} \mathrm{O}}(\mathrm{r})$ which is determined by the ratio between the thickness $\mathrm{dr}$ of a gas layer in relation to the mean free path. Integration yields the total number of collisions on the gas path taken from the liquid surface at $r_{0}$ to infinity and shows the condition of one molecular collision encounter on this way out is $1_{\mathrm{H}_{2} \mathrm{O}}\left(\mathrm{r}_{0}\right) / \mathrm{D}>1$, the well-known Knudsen condition for a free molecular beam source [2, 4] This simple model also explains, that for near surface conditions $\mathrm{l}_{\mathrm{H}_{2} \mathrm{O}} \ll \mathrm{D}$, i.e. at larger jet diameters or at higher temperatures of the liquid water and correspondingly higher jet vapor pressure, many molecular collisions occur in the outward streaming vapor and cause the onset of a supersonic jet hydrodynamic expansion into the vacuum.

The liquid jet photography shown in Fig. 1 was taken with a comparably large nominal nozzle aperture diameter $\mathrm{D}=18 \mu \mathrm{m}$ for reasons of optical resolution in this near diffraction limit of optical photography. The optically smooth, cylindrical jet propagates for a distance of $2 \mathrm{~mm}$ downstream from the nozzle exit before it starts to build up spontaneous surface oscillations and decays rapidly into a stream of fine droplets. The evaporation cooling of the jet filament amounts to $\approx 1{ }^{\circ} \mathrm{C}$ in 10 $\mu s[2,3]$. At typical jet speeds between 30 and $100 \mathrm{~m} / \mathrm{s}$ the jet surface temperature changes by only a few degrees centigrade on the first two millimeters of the smooth jet section to be employed in free vacuum experiments.

\section{Liquid Jet Flow and Decay Into Droplets}

In clearer detail liquid microjet flow contours are redrawn in Fig. 2 [3]. After leaving the nozzle channel the liquid jet free surface diameter contracts by 10 $20 \%$, depending somewhat on the nozzle shape, on the viscosity and on the surface tension of the liquid. The jet liquid flow stays strictly laminar up to very high flow velocities well above $v_{\text {jet }} \geq 100 \mathrm{~m} / \mathrm{s}$. As a consequence of the small geometrical size of the micro-jet the Reynolds numbers for flow of low-viscosity, water-like, 


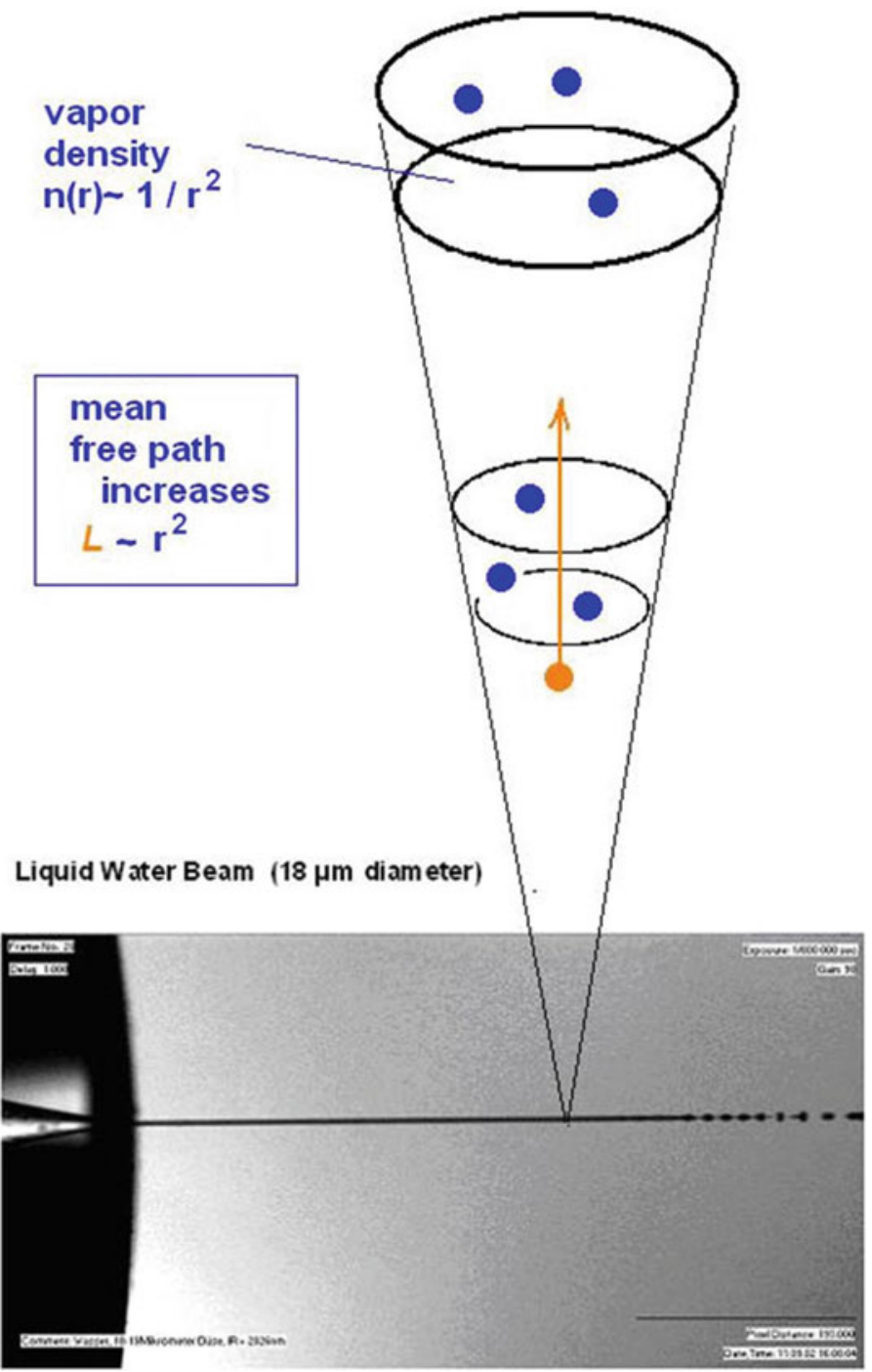

Fig. 1 A liquid microjet of water in vacuum. The photo insert shows a liquid microjet of $18 \mu \mathrm{m}$ diameter, taken at $10 \mathrm{~ns}$ exposure time. The jet emerges from a quartz nozzle, and extends for several millimeters with a smooth cylindrical envelope, before decaying into a stream of droplets. Water molecules evaporating from the liquid surface rapidly are reducing in gas density with increasing distance from the liquid filament, as is indicated by the expansion stream tube cone with two control volume disks at different distances $r$. With decreasing densities the molecular mean free path increases. Fewer than one gas collisions occur in the emerging vapor beam when the jet diameter $\mathrm{D}$ is made smaller than the vapor mean free path in equilibrium, $1_{\mathrm{H}_{2} \mathrm{O}} \approx 10 \mu \mathrm{m}$ at $\mathrm{T}=273^{\circ} \mathrm{C}$. This was Otto Stern's 'Knudsen condition' for operating molecular beam source ovens. By the intense vacuum evaporation the liquid jet cooling rate is $\sim 1^{\circ} \mathrm{C}$ in $10 \mu \mathrm{s}$, equivalent to $3{ }^{\circ} \mathrm{C}$ temperature drop on $1 \mathrm{~mm}$ length of flowing surface, for jets streaming with a velocity of $30 \mathrm{~m} / \mathrm{s}$ 


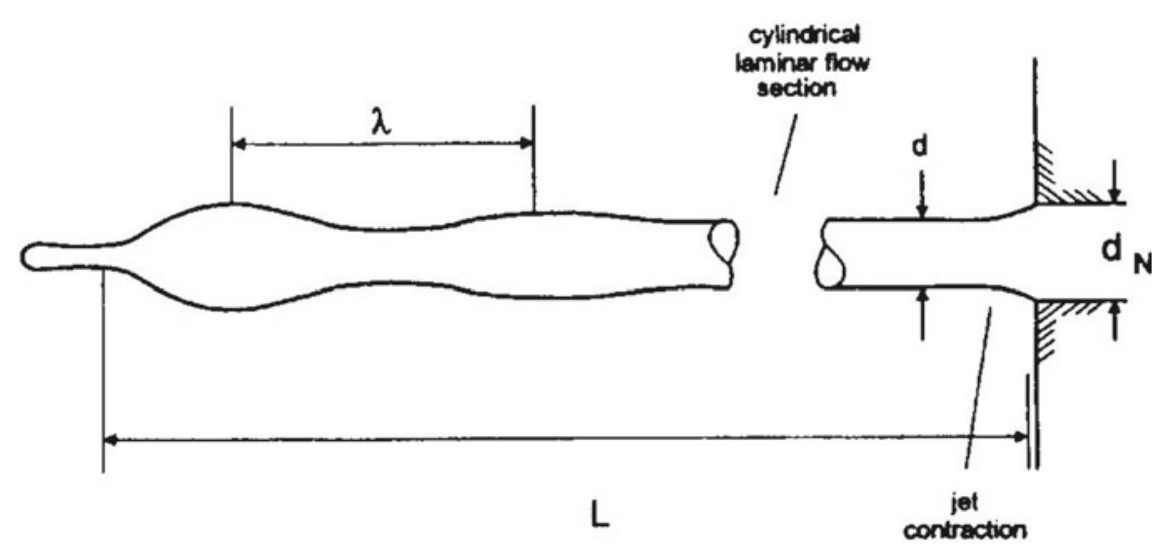

Fig. 2 Liquid jet flow and decay into droplets (figure reproduced from Ref. [3], Fig. 3)

liquids in microcapillaries are remaining well below the critical Re-values $>1500$, the limit for the onset of turbulent flow [3]. This allows the production of very fast jets with smooth surfaces and long intact cylindrical sections, with moderate jet surface cooling by the powerful vacuum evaporation. Driven by surface tension (and delayed by higher liquid viscosity) the free jet filament in some distance from the nozzle begins to form contraction ripples, spontaneously, with a wavelength $\lambda \approx$ 6-8 $\mathrm{D}_{\text {jet }}$ and then decays rapidly into a stream of approximately uniform, equidistant droplets [3]. This decay is known as the Rayleigh spontaneous decay mode for free boundary liquid filaments. Lord Rayleigh's theory shows that the decay time to form droplets for a given liquid depends on the liquids' physical properties and on the jet diameter, only (similar to the droplet dripping time from a pipette mouth). Therefore, the contiguous length $\mathrm{L}$ of the smooth cylindrical section can be extended or shrunk at will, just by changing the flow speed of the jet.

A set of experimentally determined jet length values $L$ as a function of the velocity of a liquid water jet is plotted in Fig. 3 for three different jet diameters, confirming the just discussed linear relationship between jet length $L$ and jet velocity $v_{j e t}$ due to constant jet decay time [3]. In these measurements the nozzle was illuminated from the rear side by a laser. The light beam entered the jet like a light conducting fiber; and at the breakup point at the "end" of the liquid filament the red laser light was dispersed in all directions, creating a visible red spot which could be observed easily by a remote telescope as well for a vacuum jet as in atmosphere. The jet velocity, by Bernoulli's law, is related to the square root of the nozzle pressure $\mathrm{v}_{\text {jet }}=$ Sqrt $\left(2 \mathrm{p}_{\mathrm{N}} / \rho\right)$ for a low viscosity liquid of density $\rho$. In the diagram the jet operation pressure is shown also, in the upper ordinate scale of the plot. For the smallest jets with approximately $6 \mu \mathrm{m}$ actual jet diameter, emerging from a nozzle with $\mathrm{d}_{\mathrm{N}}=$ $10 \mu \mathrm{m}$ aperture, the jet length increases linearly up to a maximum length $\mathrm{L}=4 \mathrm{~mm}$, at an approximate jet velocity near $150 \mathrm{~m} / \mathrm{s}$. At higher velocity the jet appears to decay into a diffuse, turbulent spray and the contiguous jet length begins to shorten with further increase in nozzle pressure, indicating the onset of turbulent flow and of 
Fig. 3 Jet decay length for different jet velocities and different diameters (liquid water) (figure reproduced from Ref. [3], Fig. 4)

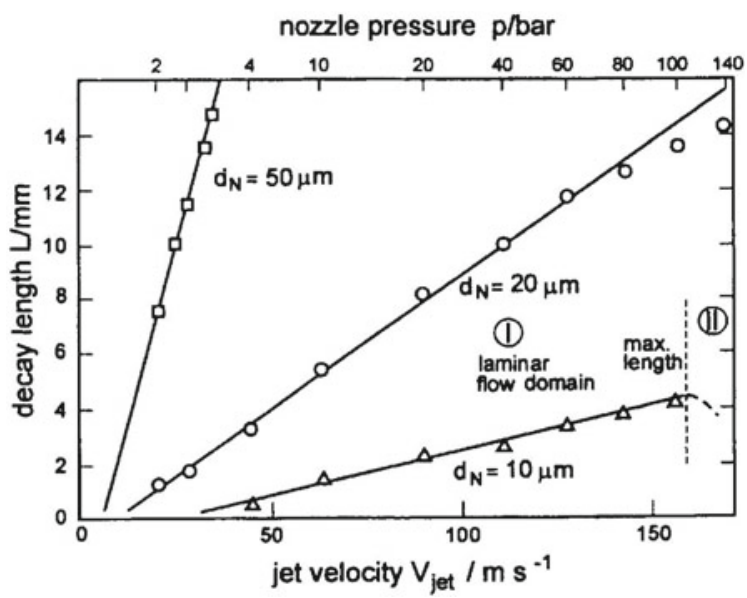

disruption instabilities by shearing forces. We can thus distinguish a laminar, smooth jet flow domain (I) for low velocities, which is well suited for surface experiments, from an unstable flow region (II) appearing above a certain critical speed. For the larger nozzle diameters $\mathrm{d}_{\mathrm{N}}=20 \mu \mathrm{m}$ and $\mathrm{d}_{\mathrm{N}}=50 \mu \mathrm{m}$ substantially longer, intact, cylindrical jet sections are obtained at much lower jet speed, as is to be expected from Rayleigh theory and its extensions, as discussed elsewhere [3, 4].

For the design of actual experiments in vacuum, microjet diameter and microjet operation conditions can be optimized and interpolated from these rough survey data in Fig. 3. From extrapolation of the jet length data shown here, it may be noted, also, that for very narrow, low viscosity water jets in the range of $1 \mu \mathrm{m}$ diameter and smaller, the maximum decay length is decreasing dramatically to less than $0.1 \mathrm{~mm}$ and shorter, limiting their value for surface probing experiments with standard size microprobe devices.

\section{Nascent Velocity Distribution of Evaporating Molecules}

The first experiment for exploring the water microjet concept in vacuum were measurements of the velocity distribution of water molecules evaporating from the liquid microjet surface, in a set-up illustrated by Fig. 4a. [2, 3]. The liquid jet here is passing in front of a skimmer collimator entrance of a molecular beam timeof-flight spectrometer. Through a $5 \mathrm{~mm}$ diameter aperture further downstream the waste water jet enters in $2 \mathrm{~cm}$ distance from the nozzle into a separate beam catcher vacuum chamber where the by then supercooled liquid droplet stream freezes as slowly growing ice-needles onto a liquid nitrogen cooled cold trap, placed in $60 \mathrm{~cm}$ distance. With vacuum pumps of several $1000 \mathrm{~L} / \mathrm{s}$ pumping speed and additional support by $10000 \mathrm{~L} / \mathrm{s}$ cryotraps a vacuum of $10^{-6}$ mbar is sustained in the main 

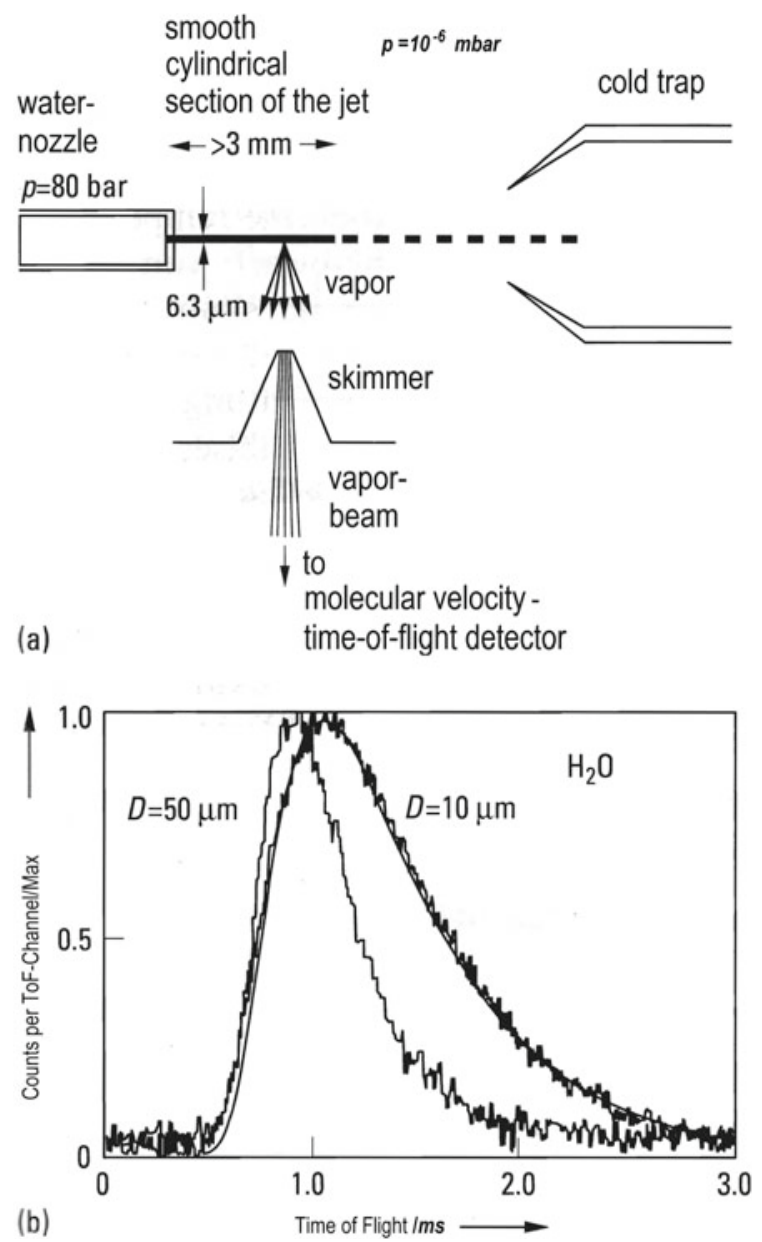

Fig. 4 Molecular beam time of flight spectroscopy of liquid microjet evaporation. a The microbeam setup shows the high-pressure nozzle, the free jet, and a beam-catcher cold trap for removal of the liquid jet. Part of the emerging vapor is extracted through an aperture in a conically shaped skimmer device. For velocity analysis a rotating disk with narrow slits is chopping the sampled molecular beam into short bursts, and molecules are detected in the ionizer of a mass spectrometer located at the end of a $0.81 \mathrm{~m}$ drift tube. $\mathbf{b}$ Two time of flight spectra of the velocity distribution of evaporated water from a thin $10 \mu \mathrm{m}$ liquid jet and from a wider $50 \mu \mathrm{m}$ jet, respectively, show a narrowed supersonic velocity distribution for the jet diameter larger than the vapor mean free path $(\mathrm{D}=50$ $\mu \mathrm{m}>\mathrm{l}_{\mathrm{H}_{2} \mathrm{O}}$ ) and, a broader Maxwell distribution (fitted by the smooth line) for collision free vapor expansion from $10 \mu \mathrm{m}$ wide liquid surface into vacuum (figure reproduced from Ref. [3], Fig. 2) 
chamber, surrounding the experimental surface probing region on the initial section of the intact, contiguous, $3 \mathrm{~mm}$ long liquid filament. For the molecular beam analysis of the vapor a sharp cone "skimmer" with $\sim 50 \mu \mathrm{m}$ entrance aperture, in two to five millimeters distance at right angle from the liquid jet propagation direction, is sampling a very small fraction of the radially evaporating, intense gas stream of water molecules. Not shown here in detail, these pass subsequently through a narrow slit in a rapidly rotating chopper wheel for producing short molecular beam bunches of $20 \mu$ s duration, and spread out in time in a drift tube on a vacuum flight path with $80 \mathrm{~cm}$ length. At the end of the drift region the dispersed molecules reach the electron bombardment ionizer region of a mass spectrometer placed in ultrahigh vacuum at pressures near $10^{-11}$ mbar for lowering the diffuse spurious gases background. By single ion counting and recording the time of ion arrival, time-of-flight spectra for the vapor molecule velocity distribution are accumulated, shown in Fig. 4b.

The two TOF spectra shown in Fig. 4b were obtained for two different sizes of liquid microjet nozzle diameter, one for $d_{N}=50 \mu \mathrm{m}$ (which is $\gg 1_{\mathrm{H}_{2} \mathrm{O}}$ ) and the other for $d_{N}=10 \mu \mathrm{m}$, respectively. The jet originating from the $10 \mu \mathrm{m}$ pinhole nozzle is contracting to $\mathrm{D}=6.3 \mu \mathrm{m}$ diameter in the free surface flow region. The velocity distribution of the evaporated water from this narrower jet surface with free evaporation Knudsen conditions $\mathrm{D}<\mathrm{l}_{\mathrm{H}_{2} \mathrm{O}}$ is very close to the theoretical Maxwell distribution which is plotted also in Fig. $4 \mathrm{~b}$, as a smooth thin line superimposed to the " $\mathrm{d}_{\mathrm{N}}=10 \mu \mathrm{m}$ " experimental spectrum. In contrast, the jet with the larger $50 \mu \mathrm{m}$ nozzle, as an example for $\mathrm{D} \gg \mathrm{l}_{\mathrm{H}_{2} \mathrm{O}}$, shows a considerably narrower velocity distribution characteristic to ongoing supersonic jet expansion, driven by multiple molecular collisions in the early phase of vapor expansion. Thus, Fig. $4 \mathrm{~b}$ gives the experimental proof that the free vacuum surface of liquid water with temperature above $0{ }^{\circ} \mathrm{C}$ can be prepared as high vacuum microjet surfaces when the jet diameter is smaller than the mean free path of the nascent vapor [2].

Numerical fitting of a Maxwell velocity distribution function to the measured distribution, in addition, yields the source temperature for the water vapor which is found to be a few degrees lower than the temperature of the nozzle and within expectations for a jet evaporational cooling model [2]. Finding a Maxwell distribution for the nascent vapor, at large, is in agreement with expectations for molecules which have to overcome a binding energy potential step barrier, i.e. the heat of evaporation, when moving from the liquid into the free vacuum space. Considering a thermal energy Boltzmann distribution in three independent cartesian coordinates, only the distribution component in the one coordinate perpendicular to the surface will be affected. Molecules will loose here the binding energy $E_{B}$ in transit through the surface dividing plane, leaving a Boltzmann distribution with reduced intensity, however, with identical temperature $\mathrm{T}$, as is easily seen by considering the mathematical separation formula: $\exp \left[\left(1 / 2 m v^{2}-E_{B}\right) / k T\right]=\exp \left(-E_{B} / k T\right) \exp \left(1 / 2 m v^{2} / k T\right)$. This noteworthy finding that the vapor temperature, in spite of the evaporation energy loss of molecules, is identical to the liquid temperature, was an often disputed, surprising fact, although it had been published as early as in 1920 in a reply of Otto Stern to comments on his earliest Molecular Beam velocity distribution measurement [5]. 
In an additional upscaling experiment with the apparatus described in Fig. 4 we tried, in vain, to observe a direct evaporation of water dimer clusters from the surface of a microjet. Dimers are well known to occur in water vapor nozzle beam jets. In a mass spectrometer ionization source the dimers fractionize into $\mathrm{H}-\mathrm{H}_{2} \mathrm{O}^{+}$ions and are expected to appear at mass 19 , next to the by far dominant water mass peak at mass 18 . Actually, a faint, distinct signal peak could be observed at mass $19,10^{-3}$ times smaller than the water monomer mass peak. However, when signal averaging the time-of flight spectrum over tens of hours at this purported peak the velocity distribution was exactly identical to the monomer peak. So it was identified to have come from the small fraction of deuterium and $\mathrm{O}^{17}$ atoms in HDO-water, and not from $\left(\mathrm{H}_{2} \mathrm{O}\right)_{2}$ water dimers with a mass of being twice the mass of monomer water that would result in average Maxwell distribution velocity smaller by a factor of $\sqrt{2}$. In conclusion, the water dimer fraction in evaporation was found to be smaller as least by a factor of five than the natural deuterium plus $\mathrm{O}^{17}$ abundance in hydrogen atom $[2,3,6]$.

In continuing the search for direct dimer evaporation from liquid surfaces, carboxylic acids were studied which are known to form strongly bound dimers in a double hydrogen bridge structure $\mathrm{COOH}_{-}$HOOC-R, with binding energies in the order of $0.3 \mathrm{eV}$ [6]. This is several times stronger than the water dimer hydrogen bond, and acetic acid microjets were found to emit large fractions, of $\geq 30 \%$, of the vapor as dimers. The vapor velocity distribution measurements of the monomer species and of the dimer species, shown in the TOF spectra Fig. 5a, b, respectively, for liquid acetic acid, $\mathrm{CH}_{3} \mathrm{COOH}$, however, show yet another, very unexpected, phenomenon: When evaluating the measured velocity distribution by fitting a theoretical distribution function, the monomer distribution, Fig. 5a is very well fitted by a Maxwellian distribution function with a source temperature of $\sim 252 \mathrm{~K}$, well in the expected range of cooling for a vacuum microjet surface temperature. The acetic acid dimer velocity distribution, Fig. 5b, in contrast, can be fitted only by a slightly supersonic "floating Maxwellian" function representing the narrower half-width-spread by a Mach number of 3-4, and yielding a total dimer molecular beam enthalpy equivalent to a dimer molecules component apparent source temperature of $365 \mathrm{~K}$ for the liquid surface. This is $100 \mathrm{~K}$ higher than the apparent monomer source surface temperature and clearly above any error bar margins [6]. This anomaly in dimer source enthalpy of liquid surface vapor sources is further confirmed by vapor velocity measurements on a liquid jet of a mixture of $20 \%$ ethanoic acid in water, shown in Fig. 5c, d. The monomer distributions of the $\mathrm{H}_{2} \mathrm{O}$ vapor component and of the $\mathrm{CH}_{3} \mathrm{COOH}$ monomers, both displayed in Fig. 5c, are well fitted by simple Maxwellian function, with practically identical liquid surface source temperatures, 281 versus $275 \mathrm{~K}$, although the average velocities of the two components differ by a factor of two, as to be expected for the mass ratio difference of 60:18 in molecular weight. This fitting result is also a proof of completely interaction-free, collisionless, vapor propagation of the two distinct mass components which show not any onset of dragging by the second component, known as the familiar seeded beams effect in more dense gas jet expansions. The dimer velocity component in this evaporating liquid mixture, in Fig. 5d, shows a slightly narrowed supersonic-like floating Maxwellian distribution 

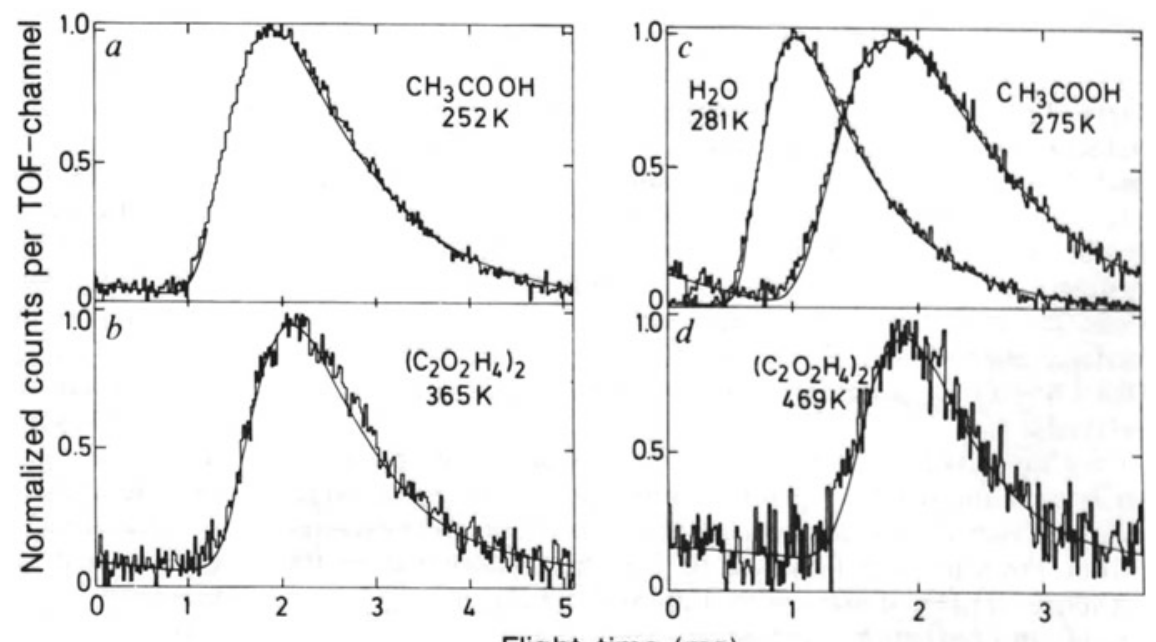

Flight time (ms)

Fig. 5 Observation of non-equilibrium evaporation of dimer molecules of pure carboxylic acid and for a mixture of $20 \% \mathrm{CHCOOH}$ in water. Temperatures shown are the (surface) source temperature calculated from the measured molecular beams time-of-flight velocity distributions. Dimers of acetic acid (Figs. 5b, d) show 100-200 K higher apparent source temperatures than the simultaneously evaporating monomers acetic acid (a) and for, both, $\mathrm{H}_{2} \mathrm{O}$ and acetic acid monomers of the mixed solution (c) (figure reproduced from Ref. [6], Fig. 2)

with much higher apparent source temperature of $469 \mathrm{~K}$, i.e., here the dimer source temperature is $200 \mathrm{~K}$ above the liquid surface temperature measured by the monomer vapor components emerging from the same liquid jet surface [6]. This astonishing anomaly is rising very interesting, far ranging questions about detailed microscopic balance of the liquid-evaporation/gas-phase-condensation process which requires the numbers of gas molecules evaporating per second and the number of molecules condensing must be in stationary equilibrium, as are to be their respective temperatures. As far as is known in statistical mechanics, or at least as far as is found in standard text books $[7,8]$, both, the distributions of evaporating molecules and the distribution of condensing molecules minus the distribution of molecules reflected from the liquid surface are all Maxwellian distributions with identical temperatures.

Although molecular liquid evaporation simulations for this phenomenon are not yet available, it was possible to give a semi-microscopic, intuitive model explanation for the observed velocity anomaly in dimer evaporation [6]: When the carboxylic dimers have formed in the liquid phase the two hydrogen bonding sites (at the $\mathrm{O}$ and at $\mathrm{HO}$ ) of the, say, acetic acid $\mathrm{COOH}$ groups are crosswise saturated toward the adjacent dimer molecule. Then it may be reasonable to assume, that the two outwardpointing hydrophobic $\mathrm{CH}_{3}$ groups act like a non-wetting nearly spherical inclusion in a water bubble or within a bubble of single hydrogen-bond, water molecules and monomerically dissolved acetic acid. When these preformed dimer inclusions approach the surface during the liquid evaporation process the bubble in this model 


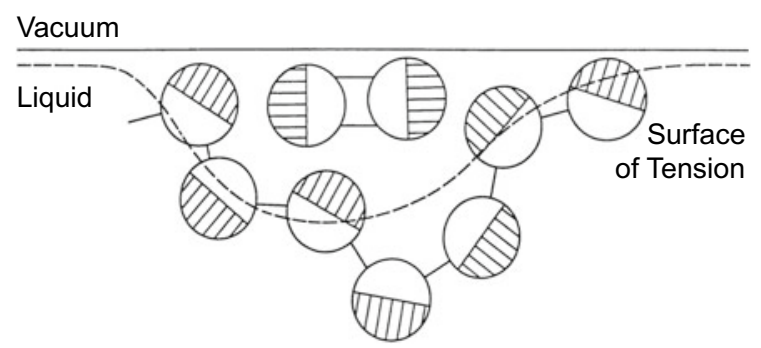

Fig. 6 Tentative surface tension model for the extra energy observed in dimer evaporation. For acetic acid $\mathrm{CH}_{3} \mathrm{COOH}-\mathrm{HOOCCH}{ }_{3}$ double hydrogen bonds form dimer inclusions in a liquid. The two $\mathrm{CH}_{3}$ groups are pointing outwards and produce a hydrophobic dimer surface. The hydrophobic dimers are ejected by surface tension with an energy proportional to the released surface tension energy of the bubble hemisphere: $\mathrm{E} \approx \sigma \pi \mathrm{R}_{2}$. Different surface tensions of acetic acid $(\sigma=1.7$ $\left.\mathrm{meV} / \AA_{2}\right)$ and of $20 \%$ acetic acid in water $\left(\sigma=3.1 \mathrm{meV} / \AA_{2}\right)$ explain readily the observed excess energies between dimer apparent source temperatures for the two liquids (figure reproduced from Ref. [6], Fig. 3)

picture will burst and the surface tension energy of the bubble will be released when the cavity is stretching out straight at the surface, transferring its freed energy like a stretched 'trampoline' to the outward moving dimer. This process is depicted in a cartoon drawing in Fig. 6. Assuming the hydrophobic dimer is sitting in a hemispherical cavity with radius of the average radius of the dimer molecule the change in surface area from hemispheric to plane, flat surface is just equal to the cross section area of the dimer molecule. With the macroscopically known surface tension value $\sigma$ the energy gain for straightening out the Surface of Tension can then be calculated as $\mathrm{E}_{\text {excess }} \approx \sigma \pi \mathbf{R}^{2}$. For tabulated [9] macroscopic surface tension values of $\sigma_{\text {Acetic }}=1.7 \mathrm{meV}^{-2}$ for pure acetic acid at $0{ }^{\circ} \mathrm{C}$ and of $\sigma_{20} \% \mathrm{Ac}=3.1 \mathrm{meV}$ $\AA^{-2}$ for a mixture of water with $20 \%$ acetic acid, it is obvious to expect a factor of two larger excess energy in the dimer evaporation from the water/acetic acid mixture, in agreement with the actually observed change of "surface excess temperature" from a value of $100 \mathrm{~K}$ in Fig. $5 \mathrm{~b}$ to $200 \mathrm{~K}$ excess in Fig. 5d. Further measurements of dimer evaporation from formic acid $\left(\sigma=2.7 \mathrm{meV} \AA^{-2}, \mathrm{~T}_{\text {excess }}=150 \mathrm{~K}\right)$ and for propionic acid confirm this model prediction of linear correlation of experimental excess dimer temperatures with the respective surface tension values [6]. The formal change of surface area calculated from the actually observed experimental value of $100 \mathrm{~K}$ for absolute cavity energy release in this crude model gives a value of $\pi R^{2} \approx 7 \AA^{2}$ model-effective geometrical cavity cross section area of the dimer $\left(\mathrm{CH}_{3} \mathrm{COOH}\right)^{2}$ molecule.

There are clearly many further unanswered phenomena left to study in evaporation of liquids, and examples of actually ongoing work on liquid surface molecular collision processes will be presented in the here following contribution, by G. Nathanson, to this Otto-Stern-Fest collection of talks. 


\section{Photoelectron Spectroscopy of Liquid Water}

The free vacuum microjet surface, equally well, is suited for electron scattering investigations and for photoelectron emission studies of liquid water, and for a majority of volatile liquid solvents in use in chemistry [10]. In particular, it has shown to be of appreciable value for providing an experimental data base of electronic structure of liquid aqueous solutions with widespread use for basic chemistry, electrochemistry, and for some studies on biological substances.

In addition to providing vacuum compatibility, here, the fast-flowing liquid jet is alleviating some of the notorious surface charging problems associated with photoemission from non-conducting materials. Charged speckles of the insulating liquid surface are just washed away, instantly, with the microjet streaming speed of several ten to hundreds of meters per second. Also, radiation heating effects and surface damage by intense photon beams are reduced by orders of magnitude by the rapid target replacement in the quasi-stationary surface of the microjet. Drawbacks and newly arising problems of the liquid jet method are: electrochemical double layer potentials build up readily near the liquid surface such as the "Stern Layer", for example, describing in greater detail electrical polarization of molecules in the surface layers of electrolytes; the moving liquid may be charged up dramatically by electrokinetic phenomena related to the internal Zeta-potential value and of the herewith associated Debye Layer thickness of the investigated liquid; in modestly well conducting electrolytic liquids external superimposed electric fields can charge up the liquid tip by current flow through the liquid filament column, leading to time dependent surface charges interdependent with the droplet decay times [3, 10]. Thus, additional measures had to be worked out for stabilizing, over the time of a photoelectron spectrum record, the electrical surface potential of poorly conducting liquid jets in order to get meaningful absolute reference potential values for measurements of photoelectron orbital binding energies from microjet photoemission spectra of aqueous solutions.

The principal construction scheme of the microjet photoelectron spectroscopy apparatus [11], shown in Fig. 7, resembles the earlier liquid jet free evaporation molecular beam sampling apparatus that was given in Fig. 1. With the extension for a photon beam directed onto the microjet on a third experimental axis, and, after replacement of the previously used molecular beam time-of-flight detector by a photoelectron hemispherical analyzer for the energy analysis, emitted photoelectrons are detected at right angle with respect to the incoming photon beam and at right angle with respect to the liquid direction. The UV and soft X-ray radiation used in photoelectron spectroscopy are strongly absorbed by gases and need the microjet vacuum for being able to penetrate the vapor shroud and to reach the liquid water surface. The mean free path of electrons in gases is one order of magnitude larger than the previously discussed free path for molecules.

For exploratory development and proofs of the technology the equipment was tested initially with a Helium-I lamp laboratory radiation source for photon energy $\mathrm{h} \nu=21.22 \mathrm{eV}$ (lambda $=58.43 \mathrm{~nm})$. And after optimization for jet and for charging 


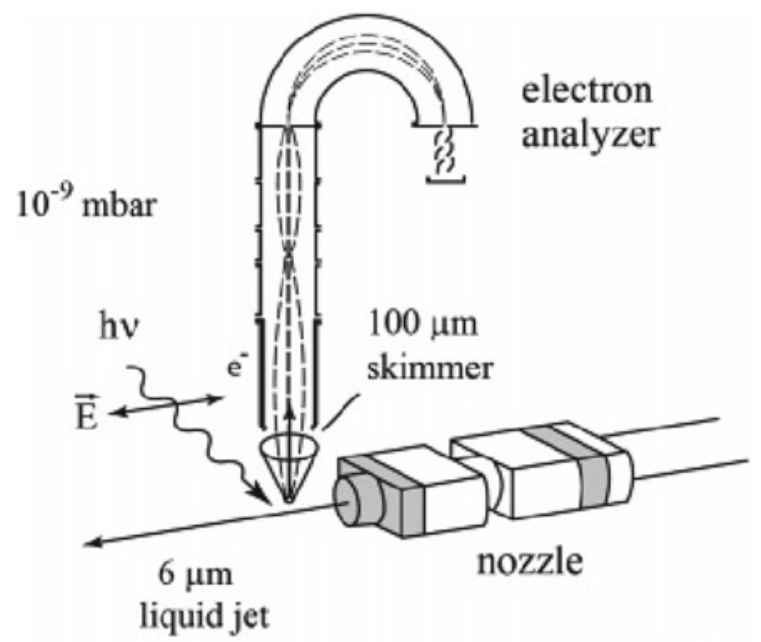

$10^{-5} \mathrm{mbar}$

Fig. 7 Photoelectron spectroscopy on a free vacuum surface of a liquid microjet of water. A focused beam of photons with defined energy $E_{p h}=h v$, ranging from vacuum ultraviolet radiation to soft $\mathrm{X}$-rays, is directed onto the microjet surface. Emitted photoelectrons are transferred into an electron energy analyzer with hemispherical electric deflection field for photoelectron spectra recording. The electron "vertical" binding energy $E_{B}$ is determining the measured electron kinetic energy $E_{\text {kin }}=$ $\mathrm{h} \nu-\mathrm{E}_{\mathrm{B}}$ (figure reproduced from Ref. [11], Fig. 18)

stability, it was providing new photoelectron binding energy spectra for the three outer valence band electrons of pure liquid water as well as for some solvents such as ethanol or gasoline, and early data for solvated halogen ions in water solutions [3, 11]. Soon after, the PES microjet apparatus could be moved to 3rd generation Synchrotron tunable radiation sources (such as Bessy II) becoming available at the end of the 1990's. These yielded for microjets photoelectron spectroscopy studies sufficiently high radiation intensities of larger than $10^{16}$ monochromatized photons per square centimeter, focused onto a perfectly microjet-suited tiny spot of size $<100$ to $<10 \mu \mathrm{m}$ [10]. The Synchrotron radiation beam outlet port, not shown here in detail in Fig. 7, is protected by a series of several narrow collimator plus vacuum pump stages, needed to separate and to protect the Synchrotron storage ring ultrahigh vacuum region, at $10^{-10} \mathrm{mbar}$, from the water vapor loaded microjet surface intersection region.

A representative, typical set of energy resolved photoelectron spectra is shown in Fig. 8. It was obtained with Synchrotron radiation photons at $\mathrm{h} v=100 \mathrm{eV}$ for salt solutions of the diatomic alkali-halide salt series CsI, KI, NaI and LiI in water [13]. The photoelectron spectrum, in a simplified point of view, is imaging the electron density of states populations in the liquid electrolyte into an energy distribution spectrum of emitted electrons. The electron orbital binding energies are determined by the difference of the incident photon energy and the measured kinetic energy of emitted photoelectrons: $E_{\text {bind }}=h v-E_{k i n}$. For the microjet spectra in Fig. 8, in molar concentrations of $2 \mathrm{~m}$ to $3 \mathrm{~m}$, one salt molecule is dissolved in 20 molecules 


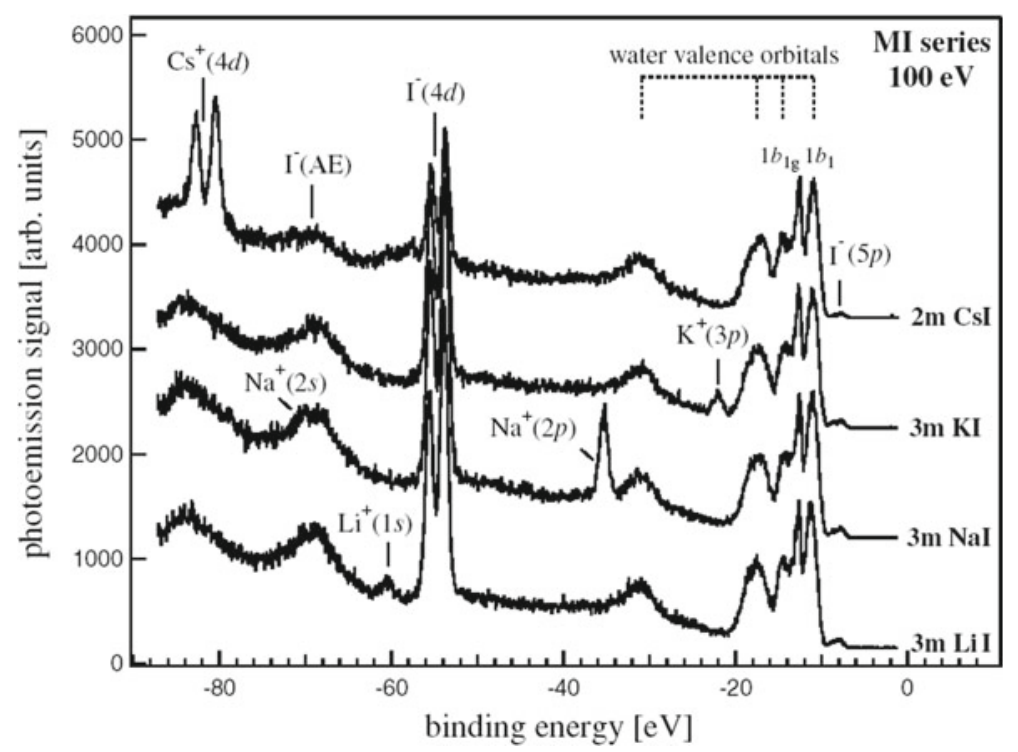

Fig. 8 Photoelectron spectra of alkali-halide salts in aqueous solution showing the valence electron states of liquid water, of the $\mathrm{I}^{-}$ion and of the anions $\mathrm{Li}^{+}, \mathrm{Na}^{+}, \mathrm{K}^{+}$, and $\mathrm{Cs}^{+}$(figure reproduced from Ref. [13], Fig. 2)

of water. At binding energies higher than the ionization onset threshold for water, near $10 \mathrm{eV}$ at the right hand side the spectra of Fig. 8, a progression of three peaks is visible which are resulting from ionization of the outermost valence orbitals of liquid water, the states designated $1 b_{1}, 3 a_{1}, 1 b_{2}$ of the water molecule and a fourth, broader peak, the inner valence orbital peak $2 \mathrm{a}_{1}$ at $32 \mathrm{eV}$ binding energy. Energy level assignments and orbital energies for the isolated water molecule are depicted in Fig. 9, together with the correlation diagram for the origin of the $\mathrm{H}_{2} \mathrm{O}$ hybridized orbitals from states of the separate oxygen and hydrogen atoms [11]. In addition to the 4 valence orbitals, the water molecule has one K-shell electron state, $1 \mathrm{a}_{1}$, at $\sim 540 \mathrm{eV}$ binding energy, to be seen later on in spectra obtained at higher photon energies. The experimental liquid water valence orbital peak energy position assignments are indicated above the valence spectrum by the horizontal scale bar in Fig. 8. In addition, in between the liquid water valence spectrum peaks, narrower faint water gas phase photoelectron peaks are visible which are caused by the vapor cloud surrounding the microjet. The most prominent gas phase spike here is designated by its gasphase peak assignment $1 b_{1 g}$ on the upper spectrum in Fig. 8 and it is shifted by approximately $1.5 \mathrm{eV}$ "gas-liquid shift" with respect to the liquid phase $1 b_{1}$ feature. During measurements, the water gas phase peak is a very helpful reference calibration point in undergoing microjet spectra evaluations. It is averaging over the electric field in the immediate surroundings of the liquid jet and, thereby, also can be used as an indicator of unintentional or unnoticed jet charging. When the potential at the jet surface is differing from the grounded chamber wall the resulting electric field 


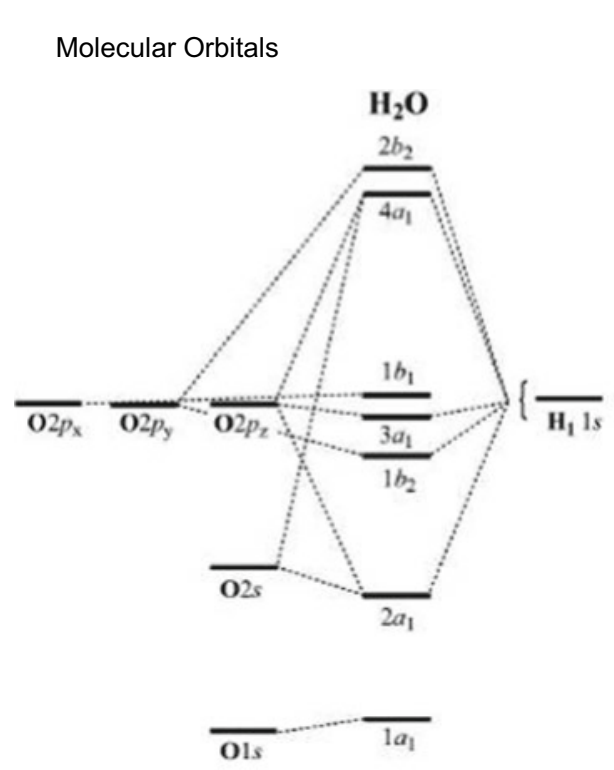

a)

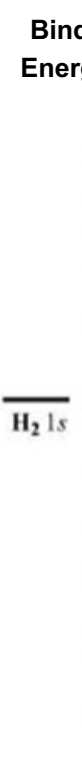

Binding

Energy / eV

$\left\{\begin{array}{l}-20 \\ -10 \\ -30 \\ -540 \\ -20\end{array}\right.$

b)

Fig. 9 a Molecular orbitals energy level diagram of the gas-phase water molecule. Intermittent diagonal lines indicate contributions of atomic orbital states of the separate $\mathrm{H}$-atoms and $\mathrm{O}$ atom to the $\mathrm{H}_{2} \mathrm{O}$ molecular orbitals. b Electron density contours for the highest occupied valence shell orbitals $1 b_{1}, 3 a_{1}, 1 b_{1}$, and the "inner valence" orbital $2 a_{1}$ (figure reproduced from Ref. [11], Fig. 20)

gradient is causing a clearly noticeable, field induced broadening of the gas phase $1 b_{1 g}$ peak and in a change of relative energy difference between liquid peak maximum and the gas phase peak.

The remarkable peak broadening of all liquid phase water valence electron energy levels with respect to the gas phase photoelectron spectrum, by amounts of the order of $3 \mathrm{eV}$ peak half width, was surprising when found in the first measurements of liquid water photoelectron spectra [10]. It appears to be associated with the multiple and rapidly varying hydrogen bonding and thus seems to reflect the heterogeneous environment of liquid water molecules [11]. However, no simple broadening explanation is visible from water simulation calculations. The numerous salt ion peaks appearing in the electrolyte spectra are notably narrower, of an order of $1.1-1.5 \mathrm{eV}$, what is probably caused by the more rigid, stable hydration shells forming in ion solvation. The $\mathrm{I}^{-}$ion in aqueous solution, for example, shows two marked photoemission peak doublets, a weak peak at 7.7/8.8 eV originating from ionization of the outermost electrons in the $\mathrm{I}^{-}(5 \mathrm{p})$ orbital of iodine and a strong (here resonance enhanced) feature for the photoelectron emission from a lower orbital, $\mathrm{I}^{-}\left(4 \mathrm{~d}_{5 / 2,3 / 2}\right)$ at ionization energy 53.8/55.5 eV. A weaker emission structure, $\mathrm{I}^{-}(\mathrm{AE})$, appearing in the spectra at the position near $70 \mathrm{eV}$ on the binding energy scale, was identified as Auger electron emission occurring when an electron hole created in the $\mathrm{I}^{-}(4 \mathrm{~d})$ 
shell by direct photoemission is filled up shortly after by one $\mathrm{I}^{-}(5 \mathrm{p})$ outer orbital electron, at the simultaneous emission of a second $5 \mathrm{p}$ electron with the excess energy difference between the $\mathrm{I}^{-}(5 \mathrm{~d})$ binding energy and the $5 \mathrm{~d} \rightarrow 4 \mathrm{~d}$ transition energy. Constant emission energy at variations of the photon energy is the experimentally easy to confirm signature for this process. The alkali-anion peak structures in the spectra allow the experimental determination of the vertical ionization energies for the outer shell electrons of the series $\mathrm{Li}^{+}(1 \mathrm{~s}), \mathrm{Na}^{+}(2 \mathrm{p})$ and $\mathrm{K}^{+}(3 \mathrm{p})$ with values of $\mathrm{E}_{\mathrm{aq}}^{\mathrm{PES}}=60.4 \mathrm{eV}$ for $\mathrm{Li}^{+}, 35.4 \mathrm{eV}$ for $\mathrm{Na}^{+}, 22.2 \mathrm{eV}$ for $\mathrm{K}^{+}$, respectively $[11,13]$. For $\mathrm{Cs}^{+}$the vertical ionization energy peak, estimated at about $15 \mathrm{eV}$, is buried in the intense liquid water valence structure peaks. The vertical binding energy values, thus obtained, are of great relevance for theoretical description of chemical processes in aqueous solution. They differ from the chemical solvation enthalpy $\mathrm{G}^{0}$ of equilibrium caloric experiments by the relaxation energies which arise by the rapid formation and realignment of the solvation shell of solvent molecules around a newly formed solvate ion species, and therefore allow an experimental determination of the important reorganization energies in electrochemical processes in polar solvents. Noteworthy, a comparison with the already known and tabulated gas phase ionization energies of the alkali and halide ions [9] shows the solvated states are changing by remarkably large amounts of energies: for $\mathrm{I}^{-}$gas phase ions the ionization energy is $3.1 \mathrm{eV}$ and shows a gas-liquid shift to $7.7 \mathrm{eV}$ in the aqueous solution experiment shown in Fig. 8. This shift is opposite in sign to the gas-liquid shift of about $12 \mathrm{eV}$ for $\mathrm{Na}^{+}$ions, decreasing from the gas phase ionization energy of $47.3 \mathrm{eV}$ to lower electron binding energy of $35.4 \mathrm{eV}$ in the liquid.

Whereas direct simulation of the peak solvation energy shifts is proofing to be demanding and time expensive, Max Born in 1922 had already proposed an elegant, very descriptive dielectric cavity (DCS) model for ion solvation which is sometimes still in use in phenomenological description of electrolytic fluids. Here the ion is thought to sit inside an empty spherical cavity inside a continuous dielectric medium with dielectric constant $\varepsilon \varepsilon_{0}$ different from the vacuum dielectric value $\varepsilon_{0}$. Using the electrostatic Maxwell equation for calculating the energy of a sphere with charge $\mathrm{Z}$ and radius $\mathrm{R}$ in an infinite dielectric medium and comparing versus vacuum with dielectric constant $\varepsilon=1$ he obtains the formula: $\Delta E_{\text {solvation }}=\left(Z^{2} e^{2} / 8 \pi \varepsilon_{0} R\right)$ $1 /(1-1 / \varepsilon)$ for the solvation energy of an ion with charge $\mathrm{Z}$ and ion radius $\mathrm{R}$, giving reasonably good numerical results for positive monatomic ions in water [10]. In the case of $\mathrm{I}^{-}$photodetachment Born-model results are far less accurate, simply because at final state with zero charge, the reorganization time of the dielectric water is difficult to include appropriately. The comparably small gas-liquid shift of $1.5 \mathrm{eV}$, only, in the ionization energy of the neutral water orbitals may also be rationalized using educated guess estimates for time-dependent dielectric constants in the DCS model. For water the static value of the dielectric constant is $\varepsilon \approx 80$. This constant is decreasing for time varying electric fields. On extremely short experimental time scales, such as the time period of light, the dielectric constant $\varepsilon$ approaches the square value of the optical diffraction index $n$ of water $(n=\sqrt{\varepsilon \approx 1.4})$ according to Maxwell's relation between the velocity of light and dielectric constant and the fact that diffraction indices are deriving from ratios of velocities of light. Therefore, for 
water dielectric reorganization on photoelectron emission with time spans of $10^{-15} \mathrm{~s}$ before the electron is distanced, the more decent estimate for the effective, dynamic dielectric response constant to the suddenly formed ion is likely to be closer to a value of $\varepsilon_{\mathrm{fs}} \approx 2$. In accord with this consideration, using the "optical" value $\varepsilon_{\mathrm{fs}} \approx 2$ in Born's DCS formula yields a gas-liquid shift of the order of $1.5 \mathrm{eV}$ for newly formed $\mathrm{H}_{2} \mathrm{O}^{+}$ions vertical ionization energies, in fairly good agreement with the observed shifts in the present water PES spectrum measurements, Fig. 8. The neutral liquid water had no time yet to respond to the appearance of the photoionized $\mathrm{H}_{2} \mathrm{O}^{+}$ion in the neutral water medium. The DCM asymptotic model is thus giving at hands an intuitive, and roughly predictive formula which should be useful when discussing the far more accurate, but, by far less transparent results of realistic computer simulations of liquids.

The measurements in a wide range of ion concentrations and for different counterions show a remarkable independence of the ionization energies which are not changing within the current experimental accuracy of $30 \mathrm{meV}$ (equivalent $3 \mathrm{~kJ} / \mathrm{mol}$ in more familiar caloric units). These vertical ionization energies, also, are not varying for different locations in the electrolyte, independent of whether the ionization takes place near the surface or in greater depth in bulk phase environment, as is demonstrated by a series of measurements on $2 \mathrm{~m}$ solutions of $\mathrm{NaI}$ with photon energies being changed between 200 and $1000 \mathrm{eV}$, in Fig. 10. Here is made use of the fact

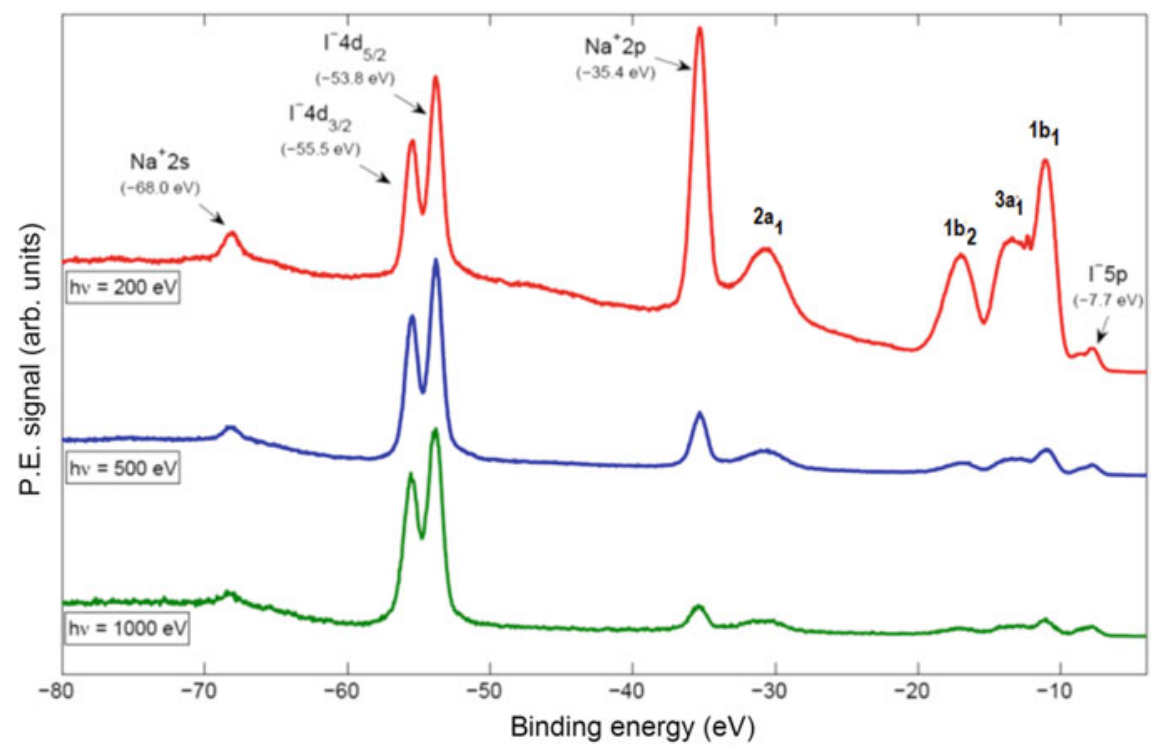

Fig. 10 Photoelectron spectrum of $4 \mathrm{~m} \mathrm{NaI}$ aqueous solution, taken at three different photon energies 200, 500, and $1000 \mathrm{eV}$. With increasing kinetic energy of photoelectrons deeper bulk regions of the aqueous solution are probed. The minimum escape depth is 2 or 3 water molecule diameters at $200 \mathrm{eV}$ electron kinetic energy, and the probing depth increases to a layer thickness of 10-12 $\mathrm{H}_{2} \mathrm{O}$-diameters for $1000 \mathrm{eV}$ photoelectron energy (figure reproduced from Ref. [14], Fig. 8) 
that for photoelectrons with different kinetic energies the escape depth for electrons increases with increasing energy [14]. The thickness of the water layer probed increases, approximately, from 3 molecular layers at $150 \mathrm{eV}(\approx 12 \AA)$ to a layer thickness of 12-15 molecular diameters of water at $950 \mathrm{eV}$ kinetic energy. The observed photoelectron signal for a given depth and given initial energy is exponentially attenuated when the photoelectrons penetrate to the vacuum space over larger distances below the surface, whereas the photoelectron energy is not altered much by electronic stopping power on these comparably short average escape path lengths. This is confirmed by the observation, that the NaI photoelectron peak shapes are not broadening with increasing photon energy, although, due to the associated higher kinetic electron energies, photoelectrons are probed for significantly greater sampling depth [14].

It may be noticed that the photoelectron spectra recorded in Fig. 10 are very smooth and show almost negligibly small statistical counting noise in comparison to the earlier measurements of Fig. 8. This is the result of continuing experimental improvements of the synchrotron radiation photon beamline and of the water photoelectron spectroscopy apparatus. The photon intensity is higher and it is focused to a smaller spot size, illuminating fully a $15 \mu \mathrm{m}$ liquid jet. In the liquid water valence peak features, therefore, the gas phase peak $1 b_{1 \mathrm{~g}}$ has vanished almost completely, and is visible only as the very weak spike residue between the $1 b_{1}$ and the $3 a_{1}$ liquid water photoelectron peaks. The three spectra are shown here with intensities normalized to the strong $\mathrm{I}^{-} 4 \mathrm{~d}$ peak feature. The wide variation of all other relative peak intensities in between the three different spectrum records is caused, primarily, by changes in photoemission cross sections for different incident photon energies.

Basically, however, the photoelectron peak intensities are proportional to absolute concentrations of molecules in the liquid target probe. Depth profile probing with different photon energies, hence, allows, also, quantitative studies of concentration changes near the liquid-gas surface of solutions. Listed ionization cross section data of reasonable computational accuracy are available by NIST [12]. Using these and the experimental apparatus functions for collection and transmission of photoelectrons it is straightforward to evaluate absolute ion concentrations for $\mathrm{Na}^{+}$and for $\mathrm{I}^{-}$from the PES measurements [14]. The thus obtained $\mathrm{I}^{-} / \mathrm{Na}^{+}$ratios over the photoelectron kinetic energy range from $\mathrm{E}_{\mathrm{kin}}=100-1000 \mathrm{eV}$, are plotted versus the electron kinetic energy, in Fig. 11. They show a clear enhancement by almost a factor of two in favor of $\mathrm{I}^{-}$anions near $200 \mathrm{eV}$ kinetic energy, sampling the composition within the first two or perhaps three water molecule diameters in the uppermost layers of the liquid. With increasing photoelectron energy the evaluated ion ratios decrease and, above $400 \mathrm{eV}$, are approaching asymptotically the value of one, expected for the bulk stoichiometry of the sodium iodide salt solution. In a computational molecular dynamics modelling study of $1.2 \mathrm{~m}$ alkali halide salt aqueous solutions (with $18 \mathrm{Na}^{+}$and 18 halogen-anion molecules in a slab of $864 \mathrm{H}_{2} \mathrm{O}$ molecules in the numerical sample calculation) the surface enhancement for halogen anions concentrations with increasing anion radii had been studied theoretically, one decade earlier [15], and can here be compared with detailed experiments. Snapshots of ion and water molecules distributions from this molecular modelling study are reproduced as a side insert in Fig. 11, adjacent to 

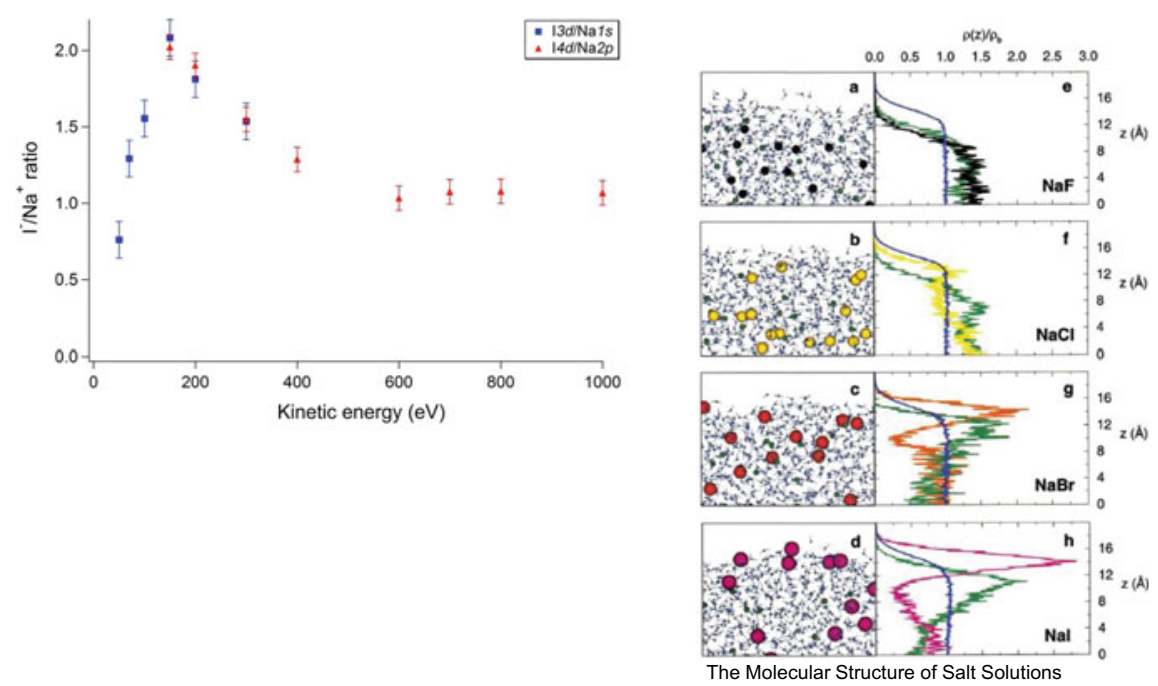

Fig. 11 (At left) evaluated anion/cation intensity ratios at different photoelectron kinetic energies show a significant propensity of $\mathrm{I}^{-}$ions over $\mathrm{Na}+$ near the surface of the $4 \mathrm{~m} \mathrm{NaI}$ aqueous solution. At electron kinetic energies larger $500 \mathrm{eV}$ the measured ratio approaches the bulk solution ratio 1:1. (At right) snapshots of computer simulations of the molecular structure of alkali-halide salts in aqueous solution show a preference for Iodine ions to the liquid-gas surface. Evaluated anion-, cation- and, water molecule-densities plotted versus the z-position coordinate of the simulation slab (Fig. 10h) are showing in quantitative detail an enhancement of the (magenta) $\mathrm{I}^{-}$anion concentration at the water surface and a tendency for immersion of $\mathrm{Na}+$ cations (red) into the bulk aqueous solution (O-atoms:blue) (figure 10 adapted from Ref. 12, Fig. 14 and Ref. 15, Fig. 1)

the measurement, and illustrate the prevalence of $\mathrm{I}^{-}$anions over the $\mathrm{Na}^{+}$cation near the model water surface [15].

\section{Core Electron Spectroscopy of Protonation/Deprotanation in Aqueus Solution}

High resolution photoelectron spectroscopy at here available energies up to $1000 \mathrm{eV}$ provides, also, a useful, sensitive, tool for chemical-environment sensitive K-shell core electron spectroscopy in a number of low- $\mathrm{Z}$ atoms, such as $\mathrm{C}$ atoms, $\mathrm{N}$, or $\mathrm{O}$-atoms which are prevailing in solute organic molecules. Chemical environment induced shifts in K-shell photoelectron spectroscopy are well known since the earliest studies of Kai Siegbahn's group in the 1960's (on solid state probes), then coined as ESCA, the electron spectroscopy chemical analysis. A great advantage of core shell spectra is in identifying chemical changes near a single, specific, atom in a chemical compound.

In Figs. 12 and 13 it is illustrated how the $\mathrm{pH}$ value induced change by protonation 
Fig. 12 Lewis structures of neutral (1a and $1 \mathrm{~b})$ and of cationic (2) imidazole. Known $\mathrm{pK}_{\mathrm{a}}=6.98$ from $\mathrm{N}^{15}$ NMR- microscale titration (Tanokura 1983) (figure reproduced from Ref. [16], Fig. 1)
ESCA spectroscopy of protonation

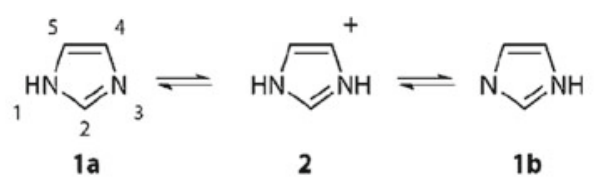

Lewis structures of neutral (1a and $\mathbf{1 b}$ ) and cationic (2) imidazole. $\mathrm{pK}_{\mathrm{a}}=6.98$ from N ${ }^{15} \mathrm{NMR}$ microscale titration, Tanokura 1983.

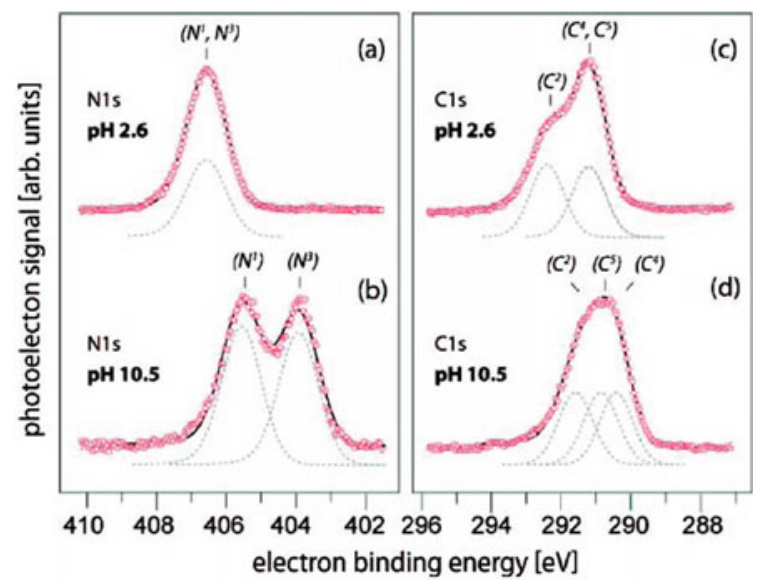

Fig. 13 Inner shell Nitrogen 1s $(\mathbf{a}, \mathbf{b})$ and Carbon 1s $(\mathbf{c}, \mathbf{d})$ photoelectron spectra show energy shifts for protonation and deprotonation of $2 \mathrm{~m}$ imidazole aqueous solutions measured at $\mathrm{pH} 2.6$ (cationic structure 2), and at $\mathrm{pH} 10.5$ (neutral molecule 1a, 1b). The photon energy is $480 \mathrm{eV}$ for the $\mathrm{N} 1 \mathrm{~s}$ measurement and $380 \mathrm{eV}$ for $\mathrm{C} 1 \mathrm{~s}$. Smooth intermittent lines show fitting results to the experimental spectra (red circles). In the protonated state the two nitrogen atoms in imidazole are indistinguishable (a) (figure reproduced from Ref. 16, Fig. 2)

can be traced on individual atoms of a solvated organic molecule [16]. Imidazole is a five atom ring compound, made of two $\mathrm{N}$-atoms and three $\mathrm{C}$-atoms, shown in the Lewis-structure representation of the molecule in Fig. 12. Protonation of the neutral dissolved imidazole molecule leads to two identical $\mathrm{NH}$ groups appear in the molecule, Fig. 12 structure (2), while the charge of the added proton is delocalized over the whole molecule. The $\mathrm{pK}$ value for imidazole protonation of $\mathrm{pK}_{\mathrm{a}}=6.98$ had been determined by, isotopically enriched, $\mathrm{N}^{15}$ NMR microscale titration by Tanokura, in 1983 [16]. The $1 \mathrm{~s}$ core electron photoelectron spectra for the N1s and for the $\mathrm{C} 1 \mathrm{~s}$ core states in $2 \mathrm{~m}$ imidazole solutions for two $\mathrm{pH}$ values, in Fig. 13, show the effects of the transition from neutral to protonated molecules. At $\mathrm{pH} 10.5$, in the lower row of spectra, the imidazole molecule is neutral and, as expected from the structure formulas 1a and $1 \mathrm{~b}$ for the neutral compound, two separate N1 s energy levels are observed for the two different nitrogen atoms in the ring, one peak for 
the nitrogen atom $\mathrm{N}^{1}$ where the hydrogen atom is attached and, separated from the first peak by $1.5 \mathrm{eV}$, a different peak $\mathrm{N}^{3}$ for the second nitrogen in position 3 of the ring (indicated in the structure drawing in Fig. 12). When changing the $\mathrm{pH}$ value both peaks are decreasing and a third peak, in the position of the N1s single peak labeled $\left(\mathrm{N}^{1}, \mathrm{~N}^{3}\right)$ in the upper spectrum is growing up in intensity. In subsequent measurement with solutions of different $\mathrm{pH}$ values, not shown here for shortness, after crossing $\mathrm{pK}=7$ value the first double peaked structure is shrinking further in amplitude until the fully protonated imidazole solution at $\mathrm{pH} 2.6$ shows only one single photoelectron peak for nitrogen, confirming theoretical chemistry results that here two pseudo-equivalent $\mathrm{NH}$ groups exist with the positive charge/electron hole-orbital distributed equally over the location of both $\mathrm{N}$-atoms, and not a $\mathrm{NH}$ group and a distinct $\mathrm{NH}^{+}$is formed after the proton attachment. This transition of the binding sites of the two distinct $\mathrm{N}$ atoms in the neutral state to two identically bound nitrogens in the protonized ion is further reflected in the $\mathrm{C} 1 \mathrm{~s}$ peak structures of the three carbon atoms bound in the molecule's ring structure. For the neutral imidazole, Fig. 13d, at $\mathrm{pH} 10.5$ a broadened carbon $\mathrm{C} 1 \mathrm{~s}$ photoelectron spectrum is observed which can be deconvoluted into 3 nearby lying $\mathrm{C} 1 \mathrm{~s}$ states with similar amplitudes, corresponding to the three different $\mathrm{C} / \mathrm{N}$ neighborhood bonding configurations of the three carbon atoms in the positions 2, 5 and 4, respectively, indicated in the, Fig. 12, structure scheme drawings $1 \mathrm{a}$ and $1 \mathrm{~b}$. In the charged state, Fig. $13 \mathrm{c}$ at $\mathrm{pH}$ 2.6, however, these carbon $\mathrm{C} 1 \mathrm{~s}$ levels contract to two overlapping states in new positions on the energy scale, as shows the deconvolution of the peak structure into two standard width peaks. The stronger peak is attributed to, both, the $\mathrm{C}^{4}$ and the $\mathrm{C}^{5}$ atoms, which are now in identical neighborhoods, as expected for the protonated imidazole species. The deconvoluted core shell $\mathrm{C} 1 \mathrm{~s}$ peak amplitudes, individually image the stoichiometric ratios for different atoms in the photoelectron spectrum and, accordingly, the joint $\left(\mathrm{C}^{4}, \mathrm{C}^{5}\right)$ carbon 1s peak shows twice the amplitude of the separate $\mathrm{C}^{2}$ peak originating from bottom $\mathrm{C}$-atom $\mathrm{C}^{2}$ connecting the two identical $\mathrm{NH}$ groups in the protonated imidazole. In summary this shows, here titration of charged/neutral molecular states can be performed quantitatively, in stoichiometric precision.

In further detail, this 1s core level PES titration demonstrates, in addition, the very distinct methodical advantage of the exceptionally high intrinsic time resolution on the order of sub-femtoseconds, given for the photoemission process by the time scale for the removal of the fast electron from the parent atom. The protonation/deprotonation bond-making and bond-breaking processes in solution take place on time scales of $10^{-12} \mathrm{~s}$. Thus, in the $1 \mathrm{~s}$ photoelectron spectra of solutions near the $\mathrm{pK}_{\mathrm{a}}$ point always two distinct peaks for protonated and deprotonated species populations appear simultaneously. In contrast in the classical NMR microtitration procedure the averaging time is limited by the period of the absorbed resonance frequency, in an order of $10^{-8}-10^{-9} \mathrm{~s}$. Therefore, averaging over many proton bond making-and-breaking cycles occurs in the NMR method which results in a frequency shift with weighted averaging over the two distinct states, only, without simultaneous separation of both levels. 
A somewhat more complex case of chemical adsorption and reaction is investigated by photoelectron spectroscopy studies shown, in Fig. 14, for analysis of the details of carbon dioxide capture in industrially used solutions of Monoethanolamine $\left(\mathrm{HOC}_{2} \mathrm{H}_{4} \mathrm{NH}_{2}\right)$ for washing $\mathrm{CO}_{2}$ from flue gas [17]. Known for more than a century, the chemical steps involved in the gas capture process have been extensively studied and characterized in great detail. 30\% monoethanolamine (MEA) in aqueous solution has a $\mathrm{CO}_{2}$ load capacity of $0.25 \mathrm{~mol} / \mathrm{L}$. The principal capture reaction is:

$$
2 \mathrm{MEA}+\mathrm{CO}_{2} \rightarrow \mathrm{MEA}-\mathrm{COO}^{-}+\mathrm{MEA}-\mathrm{H}^{+}
$$

Acid/base equilibria are:

$$
\mathrm{MEA}+\mathrm{H}_{2} \mathrm{O} \leftrightarrow \mathrm{MEA}-\mathrm{H}^{+}+\mathrm{OH}^{-} \quad \mathrm{pK}_{\mathrm{a}}=9.55
$$

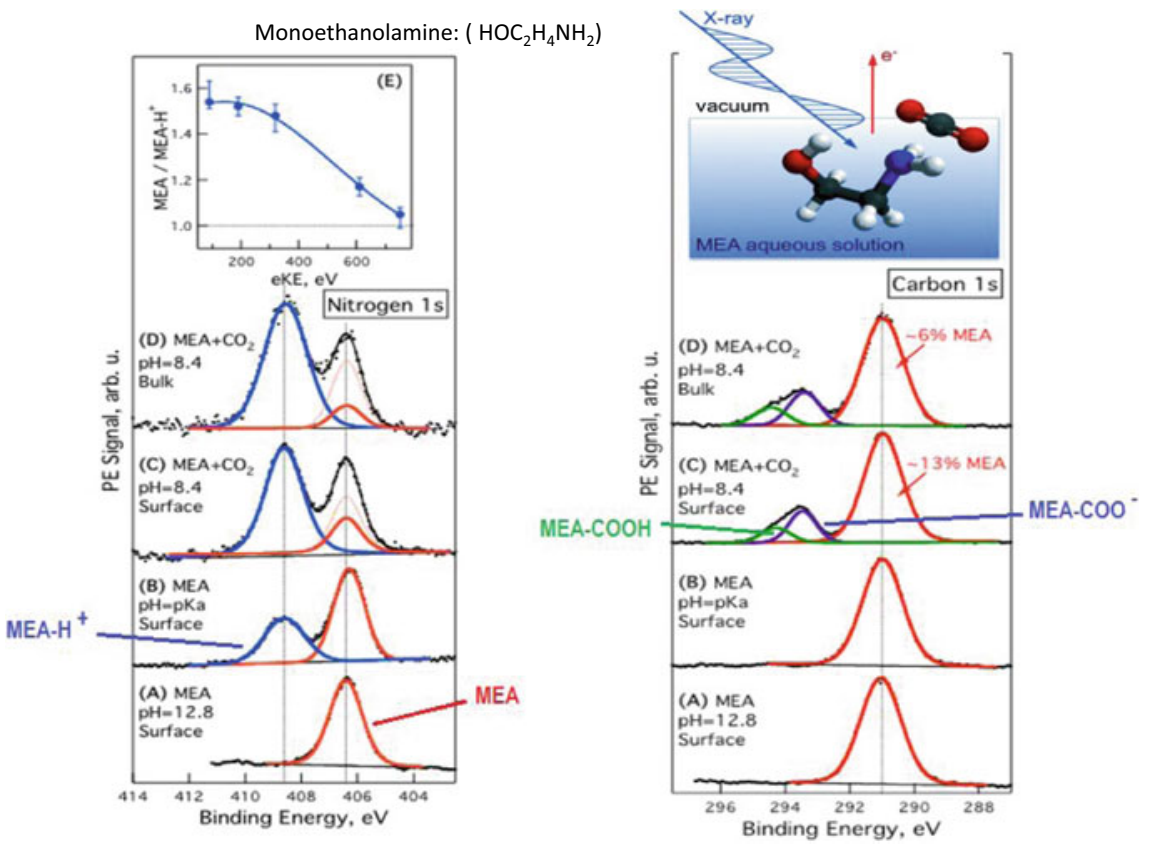

Fig. $14 \mathrm{CO}_{2}$ capture in MEA solution (Left column) nitrogen 1s photoelectron spectra for $4.9 \mathrm{~m}$ MEA (A, B) and for $\mathrm{CO}_{2}$ treated MEA solutions $(\mathrm{C}, \mathrm{D})$ show varying contributions from MEA in its neutral form (red, $406.3 \mathrm{eV} \mathrm{BE}$ ) and from protonated MEA (blue, $408.6 \mathrm{eV}$ ). Red intermittent lines represent carbamate reaction product contributions (see text). The ratio (E) of neutral and protonated MEA molecules changes as a function of depth in a solution of $\mathrm{CO}_{2}$ loaded MEA at $\mathrm{pH}$ 8.4 (Right column) carbon 1s PES spectra for MEA (A, B) and $\mathrm{CO}_{2}$ loaded MEA (C, D). In $\mathrm{CO}_{2}$ treated MEA separate peaks from carbamate (low BE, purple) and carbamic acid (green, high BE) appear. Red labels on the peaks for $\mathrm{BE}=291 \mathrm{eV}$ indicate the percentage of neutral MEA contribution (figure from Ref. [17], Figs. 1 and 2) 


$$
\mathrm{MEA}-\mathrm{COOH}+\mathrm{H}_{2} \mathrm{O} \leftrightarrow \mathrm{MEA}-\mathrm{COO}^{-}+\mathrm{H}_{3} \mathrm{O}^{+} \mathrm{pK}_{\mathrm{a}} \text { unknown (7 - 9?) }
$$

Photoelectron spectra for $\mathrm{C} 1 \mathrm{~s}$ and for N1s were taken for $\mathrm{MEA}-\mathrm{CO}_{2}$, gas loaded solutions (Fig. 14c, d) and for 30\% MEA solution without gas load (Fig. 14a, b). pH values are adjusted to the technical working point, or to other values, when needed for analysis of details. At pH 12.8 the MEA in solution is completely neutral, and the N1s binding energy spectrum (A), at the bottom line of Fig. 14, shows a single nitrogen atom peak centered at $E_{B}=406.4 \mathrm{eV}$ with half width $1.3 \mathrm{eV}$. The spectrum is a surface spectrum, taken at electron kinetic excess energy $90 \mathrm{eV}$. In (B) the $\mathrm{pH}$ value was adjusted to the (bulk) $\mathrm{pK}_{\mathrm{a}}=9.5$. In this surface spectrum a second peak, smaller by a ratio 1.6 , appears at $408.8 \mathrm{eV} \mathrm{BE}$ and is identified as the $\mathrm{N} 1 \mathrm{~s}$ signature of the protonated $\mathrm{NH}_{3}{ }^{+}$group in the MEA- $\mathrm{H}^{+}$fraction. The ratio of the two peak amplitudes represents the quantitative ratio of the two MEA species in the locally probed region of the solution. The ratio was determined in a series of additional measurements with progressively higher photoelectron excess kinetic energies in a range up to $750 \mathrm{eV}$ and shows, in plot (E) in the upper row of Fig. 14, the ratio is reducing from an excess 1.6:1 of neutral MEA-molecules near the surface to a 1:1 ratio for $750 \mathrm{eV}$ electrons which originate in greater depth from the bulk phase of the liquid.

The two remaining N1s spectra (C) and (D) are taken for gas saturated MEA solution with a $\mathrm{CO}_{2}$ load of $0.24 \mathrm{~mol} / \mathrm{L}$. These solutions change the original, equilibrium $\mathrm{pH}$ value from $\mathrm{pK}_{\mathrm{a}}$ to $\mathrm{pH}=8.4$ in the loaded state. The transformation of MEA to carbamate MEA-COO ${ }^{-}$and to carbamic acid MEA-COOH changes little in the N1s binding energy of the $\mathrm{NH}_{2}$ group on the opposite end of the molecule. The peak appearing at the position of "neutral MEA" in these spectra is the superposition of unknown fractions of contributions from loaded and non-loaded MEA with two almost identical peak shapes. More can be learned from a consideration of $\mathrm{C} 1 \mathrm{~s}$ spectra shown, adjacent to respective N1s results, on the right-hand side of Fig. 14. For neutral MEA at $\mathrm{pH} 12.8$, and for MEA at $\mathrm{pH}=9.5$ a single narrow $\mathrm{C} 1 \mathrm{~s}$ peak structure for all carbon atoms in the compound is observed at $291 \mathrm{eV}$ binding energy in the spectra shown in (A) and (B), respectively. In the $\mathrm{CO}_{2}$ saturated MEA solution at the surface (C) and for bulk solution (D) two new, distinctly visible C1s peaks arise from carbamate $\left(\mathrm{MEA}-\mathrm{COO}^{-}\right.$) and for carbamic acid (MEA-COOH), with energies shifted to higher binding energy. From the intensity ratios of the carbamate and of the carbic acid peaks, at known $\mathrm{pH}$-value of the solution, the first experimental determination of the previously uncertain $\mathrm{pK}_{\mathrm{a}}$ equilibrium with a $\mathrm{pK}_{\mathrm{a}}=8.2$ is here obtained. Further evaluation of the $\mathrm{C} 1 \mathrm{~s}$ peak ratios, in combination with measured relations between neutral and protonated species from the MEA/MEA- $\mathrm{H}^{+}$ratios of the simultaneously recorded N1s peak spectra, eventually, yields an absolute concentration ratio of (MEA) over (MEA-COO ${ }^{-}+\mathrm{MEA}-\mathrm{COOH}$ ) of $\sim 0.22$ when probing the surface and of $\sim 0.09$ for probing the bulk. The carbamate products have a preference for moving into the bulk and MEA a tendency to be enriched on the surface. This provides a perfectly cooperative cycling support for the $\mathrm{CO}_{2}$ trapping process 
at the interface and for the subsequent removal of carbamates into the bulk of the washing fluid [17].

\section{Excited States of Water, Resonant Auger Spectroscopy of $\mathrm{H}_{2} \mathrm{O}_{\mathrm{aq}}$ and $\mathrm{OH}_{\mathrm{aq}}^{-}$}

Liquid water is dissociating spontaneously into a very small fraction of $\mathrm{H}_{3} \mathrm{O}^{+}$and $\mathrm{OH}^{-}$ions, with far ranging consequences on the properties of aqueous solutions. The ions with concentrations of $10^{-7} \mathrm{~mol} / \mathrm{L}$ in pure water, by far, are too small to be observed in photoelectron spectra. Thus, solutions of strong acids $(1 \mathrm{~m} \mathrm{HCl}$, corresponding to $\mathrm{pH}=-1)$ and of strong bases $\left(\mathrm{LiOH}_{\mathrm{aq}}\right)$, instead, are to be used for photoelectron spectroscopy of the self-ionization products of water [18]. In spectra, shown in Fig. 15, valence orbitals for $\mathrm{H}_{3} \mathrm{O}^{+}$and for $\mathrm{OH}^{-}$have been studied and show a weak perturbation of the dominant $\mathrm{H}_{2} \mathrm{O}$ valence photoelectron structure, superimposed by faint photoelectron emission from $\mathrm{H}_{3} \mathrm{O}^{+}$, in Fig. $15 \mathrm{a}$, and a small peak localized at the water ionization threshold with an $\mathrm{OH}^{-}$ionization energy of $9.2 \mathrm{eV}$,

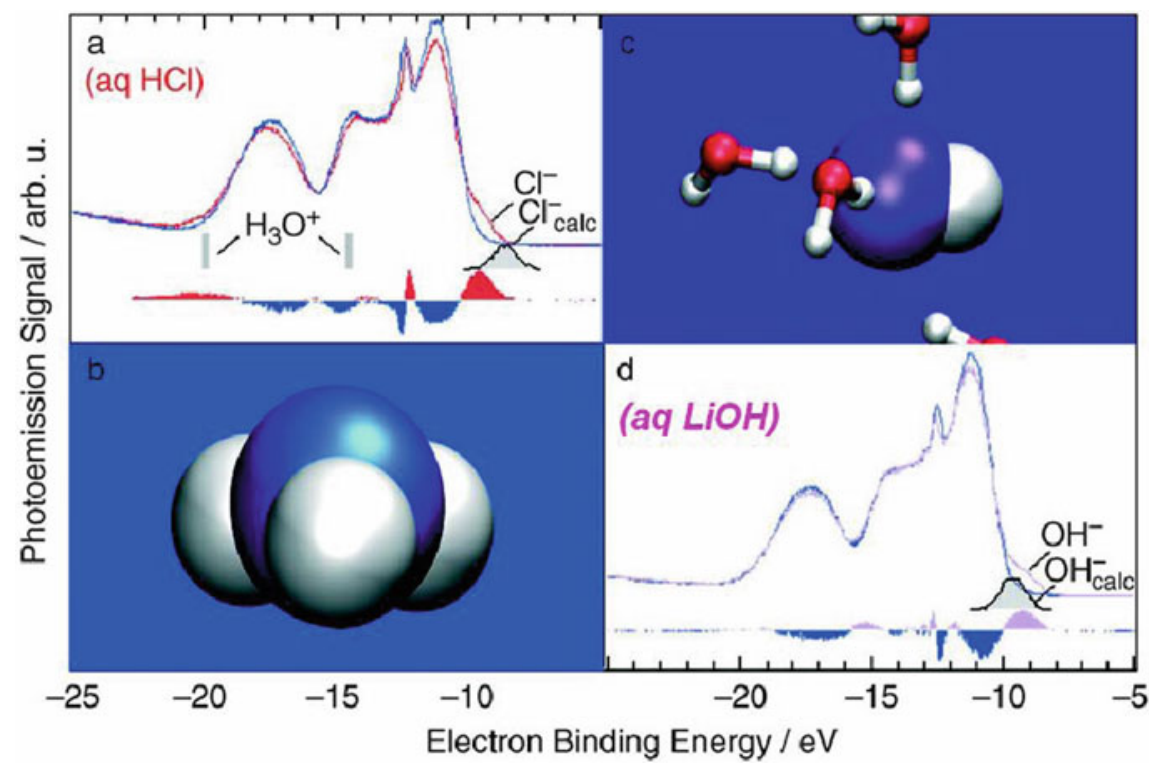

Fig. $15 \mathrm{H}_{3} \mathrm{O}_{\mathrm{aq}}^{+}$and $\mathrm{OH}_{\mathrm{aq}}^{-}$valence photoelectron spectra obtained by difference measurements of $3 \mathrm{~m} \mathrm{HCl}$ aqueous solution with neat water (a) and of a $2 \mathrm{mLiOH}$ solution (d). The $\mathrm{H}_{3} \mathrm{O}_{\text {aq }}^{+}$ion signature appears as a weak peak at $20.8 \mathrm{eV} \mathrm{BE}$, in the tail region of the $2 \mathrm{a}_{1}$ inner valence orbital of water. The $\mathrm{OH}^{-}$electron binding energy in solution is comparable to other halogen ions. Also, for comparison with the experiment, theoretically assigned ionization energy positions for $\mathrm{H}_{3} \mathrm{O}_{\mathrm{aq}}^{+}$and $\mathrm{OH}_{\mathrm{aq}}^{-}$are indicated by grey shaded features (figure reproduced from Ref. [18], Fig. 2) 
for the LiOH solution in Fig. 15d, similar in magnitude to the previously considered photoionization energies of halogen anions. Separately taken, photoelectron spectra of pure water are here compared to the photoelectron spectra of the $\mathrm{H}^{+}$and $\mathrm{OH}^{-}$in solutions and are used for extracting difference spectra shown in the lower part of Fig. 15a, d. Simulated spectra for calculated values for the $\mathrm{OH}^{-}$and the $\mathrm{Cl}^{-}$ionization potential are also shown, and are found to be in reasonable agreement with the measurement [18]. For the solvated proton different hydronium configurations were considered in the model calculation. The "Eigen"-like aqueous cation structure $\mathrm{H}_{3} \mathrm{O}^{+}$ was judged to agree best with the experimental ionization energies derived from the $\mathrm{HCl}$ solution spectra, as indicated by vertical bars for theory results, in Fig. (15a).

$\mathrm{OH}^{-}$in the gas phase exists for a single negative ion ground state, only, and similarly to the anions of halogen atoms the attractive potential well is too shallow to support any exited electronic state. In contrast, in aqueous solutions the anions are embedded in liquid water in the additional polarization potential described by the dielectric-cavity/Born-model which increases the well depth and binding energy to about $10 \mathrm{eV}$, a value large enough to allow for the existence of an excited electron state, at a binding energy of the order of 1 or $2 \mathrm{eV}$ below the ionization threshold. The optical transition to this excited state, a very strong $\mathrm{s} \rightarrow \mathrm{p}$ absorption line, was first observed in the 1930's in UV absorption spectra of $\mathrm{I}_{\mathrm{aq}}^{-}$. The phenomenon was called charge transfer to solvent (CTTS) and has long drawn the attention of spectroscopists because the CTTS states existed in liquids, only.

With the availability of narrow band, tunable soft X-ray synchrotron radiation it became possible, also, to explore this CTTS band by resonant excitation from an inner core level, and monitoring the resonance in the Auger electron emission spectrum. This has the advantage that the limitations of "classical" UV/VUV spectroscopy by the onset of the strong absorption of liquid water above 9 or $10 \mathrm{eV}$ can be offset in the Auger method. Before testing with $\mathrm{OH}_{\mathrm{aq}}^{-}$in liquid water, we explored the technology first on $\mathrm{Cl}^{-}$ions where the process is simpler and better known from previous optical VUV spectroscopy work [19]. An illustration of the different possible Auger excitation processes is shown by the three schemes drawn, in Fig. 16, for the Clanion. Direct Auger electrons (1) are emitted following an excitation of a core hole vacancy by photoelectron emission. In a rapidly following step, within a few fs, the hole in the inner shell $2 \mathrm{p}$-level is filled by an outer valence $3 \mathrm{p}$ electron and the gained energy is transferred to a second outer valence shell $3 p$ electron which is emitted as the (LMM-) Auger electron. Spectator Auger electrons (2) are emitted after resonant excitation of a 2 p electron into unoccupied levels " $\mathrm{e} 1, \mathrm{e} 2, \ldots$ " of the solvated $\mathrm{CI}_{\mathrm{aq}}^{-}$ion. The energy of the spectator-Auger electron is higher than for normal Auger electrons because the originally photon-excited electron is still present in the ion and increases the coulomb forces acting on the outgoing Auger electron. A third process may occur (3) in a shake down, transferring electron-energy of the resonantly populated levels to other internal states before the Auger electron is emitted, with the result of an additional change in the kinetic energy of the emitted spectator-Auger electron.

In a series of photoelectron spectra records at closely spaced energies of the incoming synchrotron radiation, tuned over the region of interest for expected $2 p$ to CTTS transition, shown in Fig. 17, these discussed Auger phenomena can be 
$2 p$ core-hole excitation of the chloride anion, $\mathrm{Cl}^{-}(\mathrm{aq})$

(a)

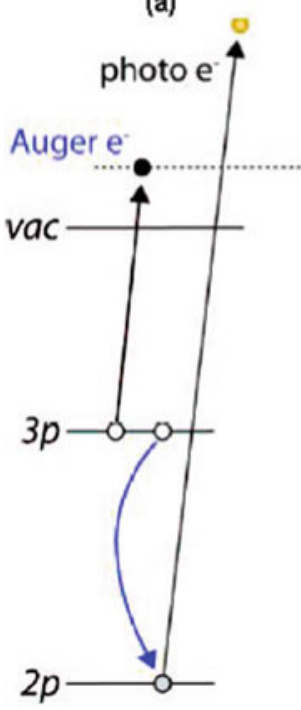

$1 \mathrm{~h}_{\mathrm{c}} \rightarrow 2 \mathrm{~h}$

Direct

Auger (b)

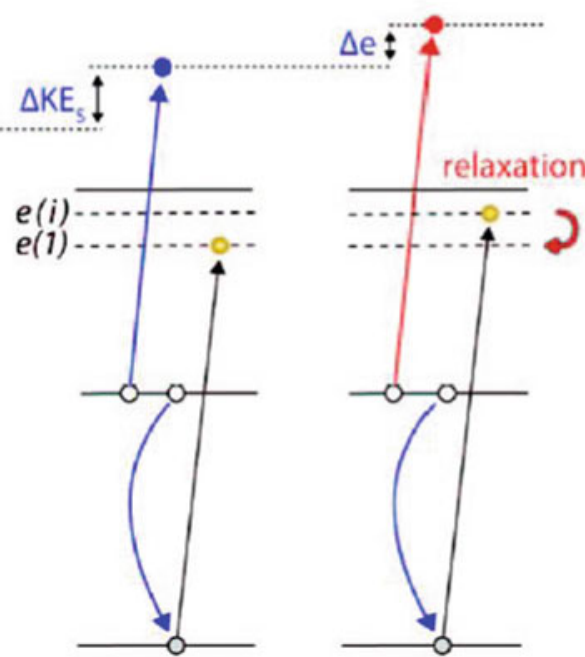

$$
\begin{aligned}
& 1 \mathrm{~h}_{\mathrm{c}} 1 \mathrm{e}(\mathrm{i}) \rightarrow \\
& 2 \mathrm{~h}_{\mathrm{v}}^{\mathrm{c}} 1 \mathrm{e}^{\prime}(\mathrm{i})
\end{aligned}
$$

Spectator Auger after resonant excitation (c)

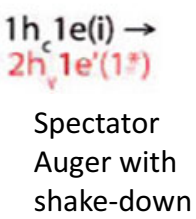

Fig. 16 Auger processes associated with $2 \mathrm{p}$ core-hole excitation of the chloride anion, $\mathrm{Cl}_{\mathrm{aq}}^{-}$, in aqueous solution. Negative halogen ions have no excited states in the gas phase. In aqueous solution one or several new excited states appear, called charge transfer to solvent states (CTTS). a Direct Auger process with emission of a photoelectron followed by emission of the Auger electron. $\mathbf{b}$, c Spectator Auger emission after resonant excitation of one of the CTTS states e(1) - e(i). The spectator Auger electron kinetic energies are shifted due to electrostatic interaction by the presence of the resonance electron. c Occasionally, energy of the resonantly excited state is transferred to nearby internal states before the Auger electron is emitted (figure reproduced from Ref. [19], Fig. 5)

actually observed [19]. At the lowest photon energy, at $200 \mathrm{eV}$, the electron kinetic energy spectrum shows only the familiar peak structures of the liquid water valence bond states, and in addition, the small $\mathrm{CI}_{\mathrm{aq}}^{-}$peak shoulder adjacent to the right-hand side of the water $1 b_{1}$ peak. At the highest photon energy 204.8, the electron kinetic energy spectrum of the water valence structure photoelectron spectrum is shifted toward $4.8 \mathrm{eV}$ higher kinetic energies in accordance with the higher photon energy. In addition, the $204.8 \mathrm{eV}$ spectrum shows the strong, fully developed, regular Auger peak for $\mathrm{CI}_{\mathrm{aq}}^{-} \mathrm{LMM}$ Auger emission with indicated doublet splitting n, $\underline{\mathrm{n}}$. The LMM Auger peak starts to develop at photon energies larger than the $2 \mathrm{p}_{1 / 2,3 / 2}$ level ionization energy of $201 \mathrm{eV}$. Its signature is the constant kinetic electron energy, independent from the incident photon energy. Most importantly, however, in the intermediate 
Fig. 17 Photoelectron and Auger electron spectra for $\mathrm{Cl}_{\mathrm{aq}}^{-}$measured at photon energies near the detachment energy for $2 \mathrm{p}$ orbital electrons. A $3 \mathrm{~m} \mathrm{LiCl}$ aqueous solution liquid jet is used. Normal Auger peaks for the $\mathrm{Cl}_{\mathrm{aq}}^{-} 2 \mathrm{p}_{3 / 2}(\mathbf{n})$ and $2 \mathrm{p}_{1 / 2}$ (n_) LMM process (see Fig. 16a) occur at constant kinetic energy. Emitted photoelectron peak positions for water move with increasing photon energy. Resonant absorption transitions into unoccupied CTTS states of $\mathrm{Cl}_{\mathrm{aq}}^{-}$are readily observable at the resonant Auger line positions 1_, 2, 2_, 3_, 4. Resonance features 2,4 are attributed to transitions originating from $\mathrm{p}_{3 / 2}, \mathbf{1}_{-}, \mathbf{2}_{-}$and 3_from $\mathrm{p}_{1 / 2}$ Auger resonances in CTTS state (figure reproduced from Ref. 19, Fig. 2)

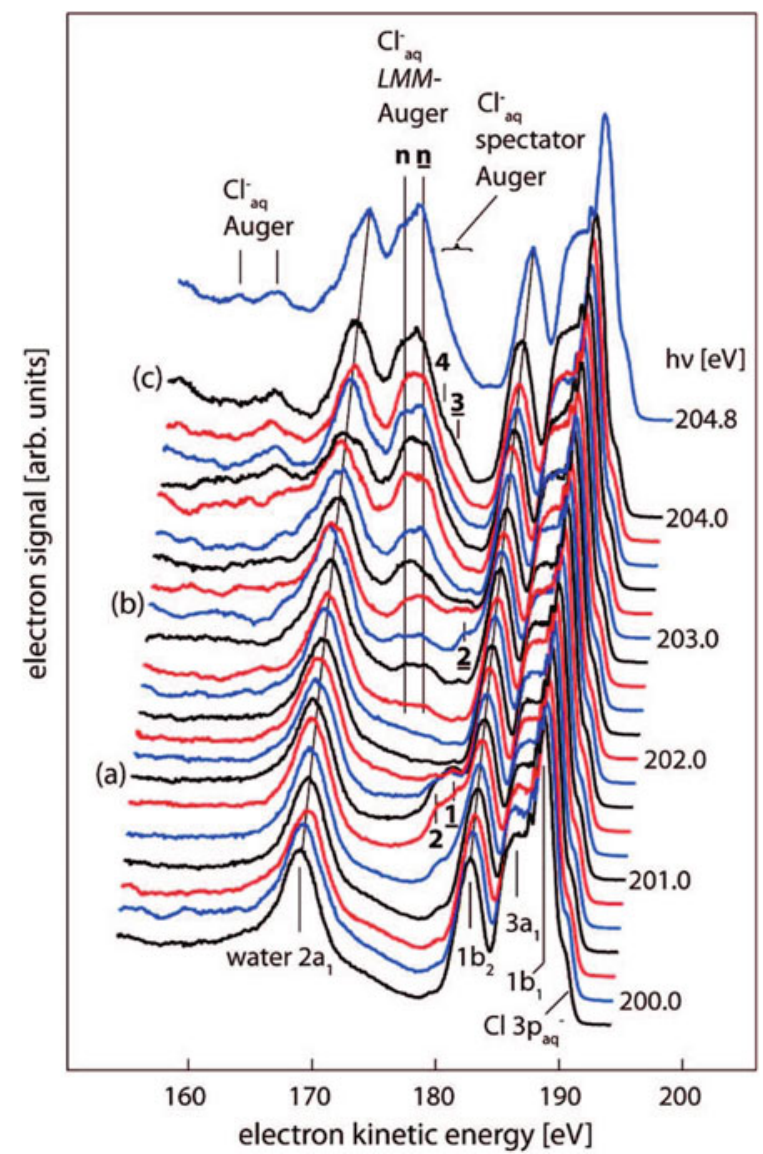

region of scanned photon excitation energies some photoelectron spectra show small intensity bumps appearing only in very narrow excitation energy ranges $\leq 0.3 \mathrm{eV}$ full half width, which originate from the searched-for resonant excitation of $\mathrm{Cl}^{-}$ unoccupied level states. The resonance Auger features are identified in Fig. 17 by the numbers $1,2, \underline{3}$ and 2 or 4 , pointing to the observed features at three different excitation photon energies where resonances could be detected. The region of the $(2,1) \mathrm{CI}_{\mathrm{aq}}^{-}$spectator-Auger peaks group is drawn enlarged in Fig. 18, showing the signal evolution as a function of photon energy. The blue peaks are Gaussian fits of 2 and 1 to the experimental kinetic energy spectra envelopes. After analogous evaluation of all other observed spectator-Auger resonances an energy level diagram of these newly identified unoccupied electronic levels can be constructed and is shown in Fig. 19 together with the also determined absolute energy level values for the occupied electron orbital states $3 p$ and $2 p_{1 / 2}, 2 p_{3 / 2}$ which were obtained simultaneously from the measured photoelectron spectra of $\mathrm{CI}_{\mathrm{aq}}^{-}$. The $\mathrm{Cl}^{-}$excited states orbitals in aqueous solutions are here found at binding energies of 2.5 and 
Fig. 18 Enlarged region of $\mathrm{CI}_{\mathrm{aq}}^{-}$photoelectron spectra in Fig. 17, showing the signal evolution of spectator

Auger-electron peaks 2 and 1 _ at photon energies

between $200.4 \mathrm{eV}$ and 201.4 $\mathrm{eV}$. Blue peaks are Gaussian fits to structures $\mathbf{2}$ and $\mathbf{1}_{-}$in the experimental photoelectron spectra (red) (figure reproduced from Ref. 19, Fig. 3)

Fig. 19 Experimental CTTS states of Cl-aq and electron binding energies for the $3 p$ and $2 \mathrm{p}$ states of the negative chlorine ion in $3 \mathrm{~m} \mathrm{LiCl}$ aqueous solution (figure reproduced from Ref. 19, Fig. 6)
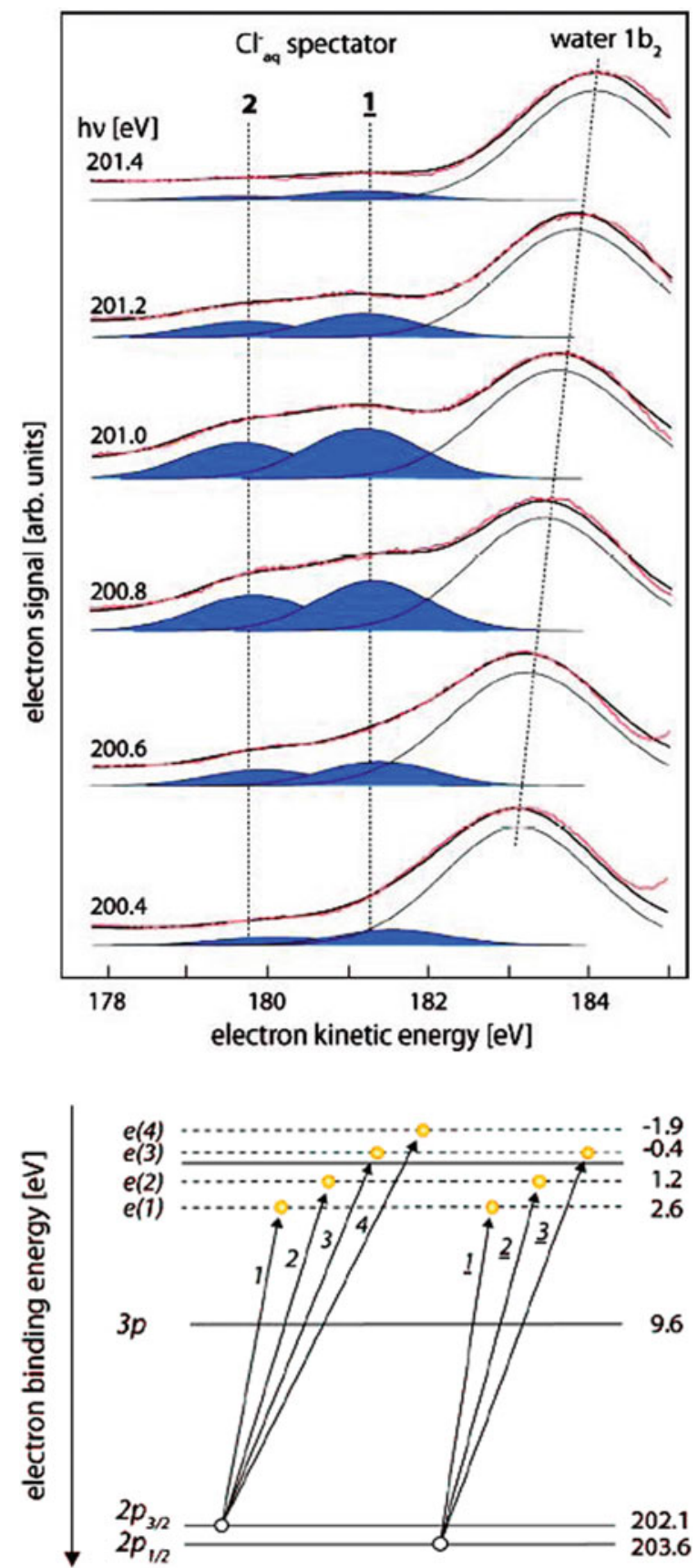
of $1.3 \mathrm{eV}$, and two further, antibound state resonances were identified, appearing slightly above the vacuum level, at -0.4 and $-1.9 \mathrm{eV}$, respectively [19].

With the expertise acquired in resonant spectroscopy of CTTS states in the simple, spherical $\mathrm{CI}_{\mathrm{aq}}^{-}$anion, we return to the $\mathrm{OH}_{\mathrm{aq}}^{-}$with a study to find the CTTS state here in resonant excitation from the $1 \mathrm{~s}$ core state of the $\mathrm{O}$-atom in $\mathrm{OH}_{\mathrm{aq}}^{-}$which is the only inner shell state available in this anion entity [20]. Fig. 20 shows electron kinetic energy spectra obtained in a narrow scan range of photon energies near the expected O1s to CTTS transition. In a series of preceding photoelectron experiments the $\mathrm{OH}_{\mathrm{aq}}^{-}$ O1s binding energy had been determined to be $536.0 \mathrm{eV}$ (shown in Fig. 21, in a combined experimental energy level diagram for liquid $\mathrm{H}_{2} \mathrm{O}$ and aqueous $\mathrm{OH}^{-}$). In search for the $1 \mathrm{~s}$ resonance of $\mathrm{OH}_{\mathrm{aq}}^{-}$near the continuum threshold, kinetic energy electron spectra are shown, in Fig. 20, for $4 \mathrm{~m} \mathrm{NaOH}$ solutions (red lines) and for

Fig. $204 \mathrm{~m} \mathrm{NaOH}$ aqueous solution photoelectron spectra, showing resonant Auger-electron spectra (b, c) at 532.2 and $532.8 \mathrm{eV}$, and (ultrafast) intermolecular coulombic decay of $\mathrm{OH}_{\mathrm{aq}}^{-}$. The photon energy is scanned near the ionization threshold for the oxygen $\mathrm{O} 1 \mathrm{~s}$ inner shell orbital. In addition, photoelectron spectra of pure water are recorded (blue) for reference. Resonance Auger electron peaks 2, 3, and 4 reveal the existence of a very fast energy transfer process from excited $\mathrm{OH}^{-}$to the $\mathrm{H}_{2} \mathrm{O}$ solvent. The small peak at highest kinetic energy, far right, arises from $\mathrm{O} 1 \mathrm{~s}$ ionization by spurious, second harmonics photons with energy $2 \mathrm{~h} v$, and, provides a method for highly accurate absolute energy calibration (figure reproduced from Ref. [20], Fig. 1)

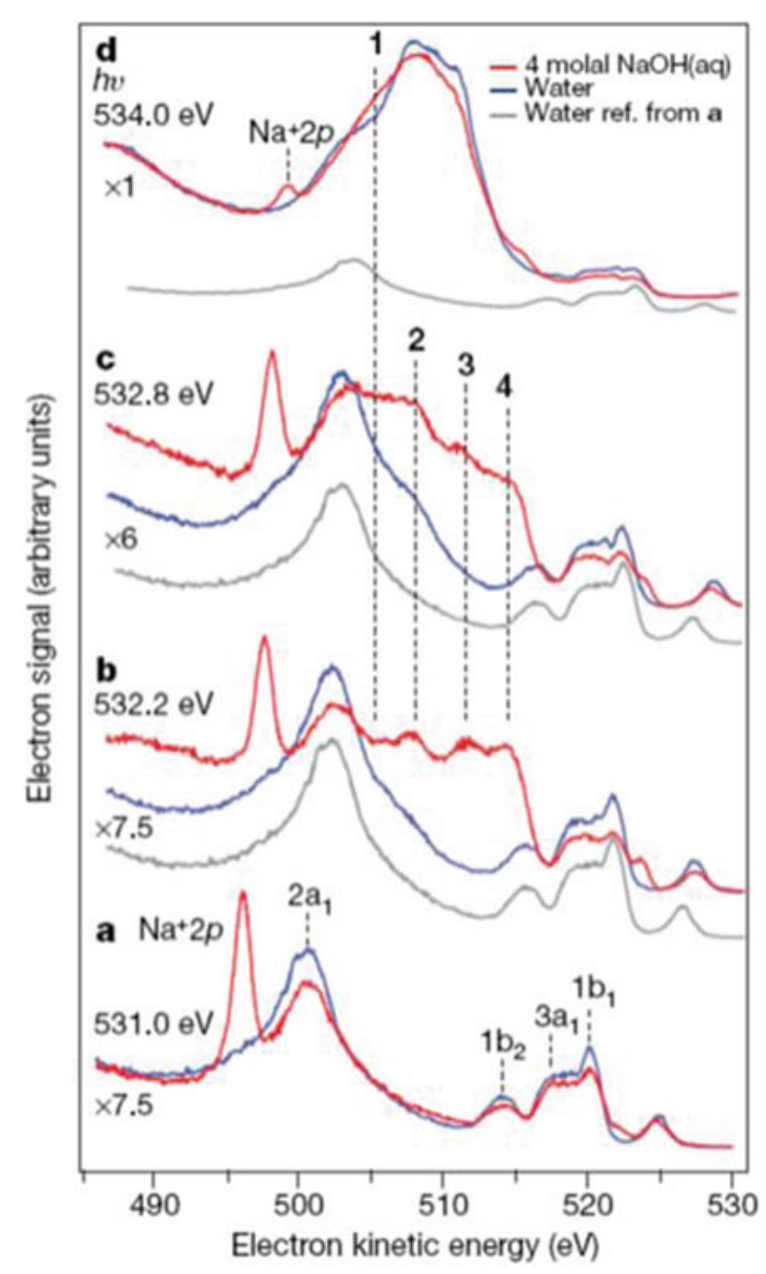




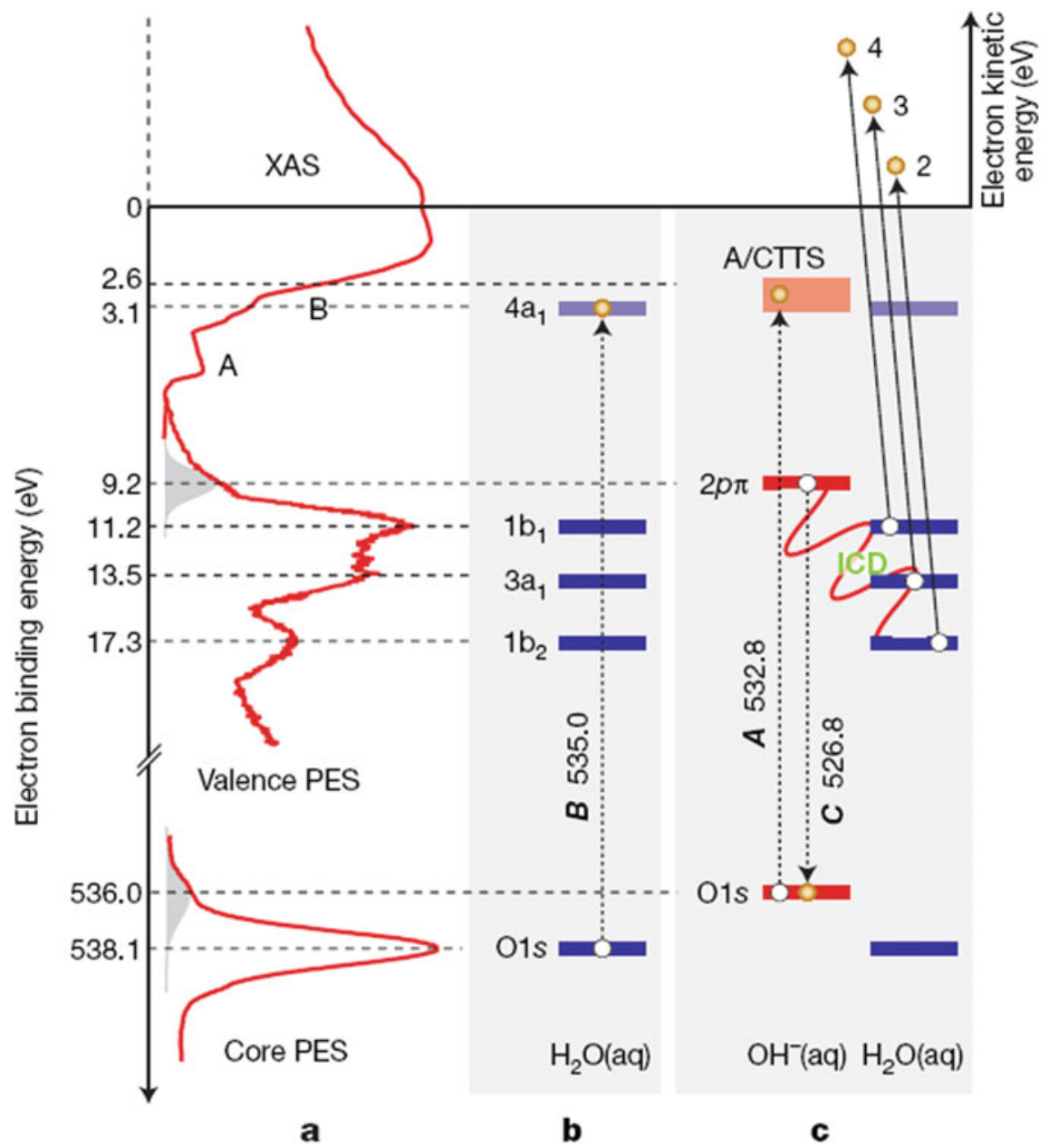

Fig. 21 Complete, experimental energy level diagram of $\mathrm{OH}_{\text {aq }}^{-}$and $\mathrm{H}_{2} \mathrm{O}_{\text {aq }}^{-}$obtained from liquid microjet photoelectron spectroscopy of a $4 \mathrm{~m} \mathrm{NaOH}$ solution. Shown are bound states and, in addition, un-occupied energy levels $\left(4 \mathrm{a}_{1}\right.$ of $\mathrm{H}_{2} \mathrm{O}$ and a CTTS of $\mathrm{OH}_{\mathrm{aq}}^{-}$) identified by resonant Auger spectroscopy. Plotted on the left side (a) are experimental PES spectra used for the energy level determination; shaded Gaussian peaks (light grey) are the deconvoluted contribution of $\mathrm{OH}_{\mathrm{aq}}^{-}$ states (figure reproduced from Ref. [20], Fig. 2)

neat water (blue line) at the 4 photon energies 531.0, 532.2, 532.8, and $534.0 \mathrm{eV}$. Figure 20a, at $531.0 \mathrm{eV}$ photon energy, shows a reference spectrum taken at an energy slightly below the onset of resonances. In the (blue line) neat water valence spectrum the well known four peaks $2 a_{1}, 1 b_{2}, 3 a_{1}, 1 b_{1}$ are observed and the (red line) $\mathrm{NaOH}_{\mathrm{aq}}$ spectrum is showing water plus the additional $\mathrm{Na}_{\mathrm{aq}}^{+} 2 \mathrm{p}$ lines as well as the small valence $\mathrm{OH}_{\text {aq }}^{-}$peak with $\mathrm{E}_{\text {kin }}=521.8 \mathrm{eV}$. At the subsequent photon energy values at $532.2 \mathrm{eV}$ and at the close by value $532.8 \mathrm{eV}$ the $\mathrm{NaOH}$ brine spectra (red) have 
changed dramatically from the, respective, neat water photoelectron spectra (blue), showing $\mathrm{O} 1 \mathrm{~s}$ resonance spectator-Auger features in a remarkably high intensity and with very broad additional structure. Further scans for a slightly higher photon energy $534.0 \mathrm{eV}$, in Fig. 20d, show this is off resonance, already. Except for the $\mathrm{OH}^{-}$peak and the $\mathrm{Na}^{+}$, there are no big differences left between the recorded neat water and the $\mathrm{NaOH}$ spectrum. A broad and intense peak structure beginning to appear in, both, neat water and in $\mathrm{NaOH}$ solution in Fig. 20d arises from a superposition of the water valence state $2 \mathrm{a}_{1}$ photoelectron emission peak and an onset of strong water Auger peak structure resulting from excitation of the $4 \mathrm{a}_{1}$ unoccupied state of $\mathrm{H}_{2} \mathrm{O}_{\mathrm{aq}}$ from $\mathrm{O} 1 \mathrm{~s}$ with excitation centerline energy $535.0 \mathrm{eV}$. It is designated by the label 1 on top of the spectrum. Within the spectacular $\mathrm{OH}^{-}$spectator resonance spectra, Fig. 20b, c, three adjacent peak features are recognized with spacings closely matching the energy differences and overall structure between the $1 b_{2}, 1 a_{1}$ and $1 b_{1}$ valence photoelectron spectra of $\mathrm{H}_{2} \mathrm{O}$. They are marked by the letters 2,3 and 4 and three additional vertical lines, for underlining the fact that the kinetic energies are constant for Auger electron emission. The apparent, unexpected mixing of valence states of adjacent $\mathrm{H}_{2} \mathrm{O}$ molecules into the Auger decay process of core hole excited $\mathrm{OH}^{-}$is an obviously new phenomenon, difficult to understand [20]. Although it is well known, that the high electrical mobility of the $\mathrm{OH}^{-}$ion in liquid water has to be attributed to a charge migration process, rather than to molecular diffusion, the time scale is $10^{-12} \mathrm{~s}$ for the established charge migration model where a local $\mathrm{OH}^{-}\left(\mathrm{H}_{2} \mathrm{O}\right)_{\mathrm{n}}$ hydration cluster is rearranging bonds and hopping the charge to a newly formed $\mathrm{OH}^{-}$ center. This mechanism, therefore, must be disputed as being very unlikely because the charge migration step takes orders of magnitudes longer than the decay time of $7 \mathrm{fs}$, available for the here observed O1s Auger processes. A different, more recent theory attributes the mixing of adjacent molecules' molecular states in an Auger process to a very rapid Intermolecular Coulombic Decay (ICD) phenomenon, with quantitative details for $\mathrm{OH}_{\mathrm{aq}}^{-}$resonance here also still open to discussion.

With the tentative assignments of the $\mathrm{OH}_{\mathrm{aq}}^{-}$resonance emission peak structures number 2, 3 and 4 to Auger emission of water valence electrons $1 b_{1}, 2 a_{1}, 1 b_{2}$, in Fig. 20b, c, the picture emerges that one electron from the $\mathrm{OH}_{\mathrm{aq}}^{-}$valence state $2 \mathrm{p} \pi$ is filling the $\mathrm{O} 1 \mathrm{~s}$ hole in $\mathrm{OH}_{\mathrm{aq}}^{-}$and, simultaneously, in undisclosed dynamical detail, one of the neighbor- $\mathrm{H}_{2} \mathrm{O}$ valence electrons is emitted and observed in the recorded kinetic energy electron spectra. This Auger process interpretation is symbolically depicted in the energy level diagrams at the right-hand side in Fig. 21c. In this energy level scheme are compiled all experimentally determined energies for $\mathrm{OH}_{\mathrm{aq}}^{-}$ and, also, all levels for liquid $\mathrm{H}_{2} \mathrm{O}$ (in Fig. 21b) which have been obtained from the here described photoelectron spectroscopy measurements. On the left-hand side of the level diagram, Fig. 21a, characteristic photoelectron spectra traces for core and valence states of water and of $\mathrm{OH}^{-}$in solution are displayed for illustrating the relationship between measured PES features and the herewith determined electron orbital energies in aqueous solution. The also shown energetic positions of the unoccupied excited state $4 a_{1}$ of water in aqueous solution and the new CTTS state for 
solute $\mathrm{OH}^{-}$ions are determined with the resonant Auger spectroscopy method, just discussed.

For comparison with more traditional and longer established XAS methods, in the upper part of Fig. 21a is shown, furthermore, a measurement of electronic structure in liquids by X-ray absorption spectroscopy on the K-edge, in the region of O1s excitation to the ionization vacuum level of an aqueous $\mathrm{NaOH}$ solution. In the $\mathrm{X}$ ray near edge absorption scan with high resolution tunable x-ray radiation, here the total absorption increases in a large step at the K-edge of water. In addition, pre-edge absorption XAS structures appear at slightly lower photon energies, assigned with the letters $\mathrm{A}$ and $\mathrm{B}$ and originating from resonance absorptions from unoccupied bound states. The pre-edge structure peak $B$ is related to the excitation of the $4 a_{1}$ unoccupied state of water at $3.1 \mathrm{eV}$ electron binding energy according to the photoelectron energy absolute calibration in Fig. 21b. The peak A observed for $\mathrm{OH}^{-}$in the XAS spectrum appears at lower excitation energy and its position can be interpreted correctly, only, after it is known from PES results in Fig. 21c that the vertical ionization energy of $\mathrm{O} 1 \mathrm{~s}$ electrons of the $\mathrm{OH}_{\mathrm{aq}}^{-}$with $536.0 \mathrm{eV} \mathrm{BE}$ is smaller by $2.1 \mathrm{eV}$ than the liquid water $\mathrm{O} 1 \mathrm{~s}$ ionization energy value. Not visible at all in XAS, of course, is the Auger decay transfer from $\mathrm{OH}^{-}$to vicinal $\mathrm{H}_{2} \mathrm{O}$ molecules, following excitation of CTTS state.

\section{Concluding Remarks}

In going through this retrospective on the development of liquid water microjets for molecular beam studies of evaporating nascent molecular velocity distributions, and then extended, for X-ray photoelectron spectroscopy as diverse as simple determination of valence energies in electrolytes, concentration measurements of surface versus bulk abundance, diagnostics of $\mathrm{pH}$ sensitivity of protonation-deprotonation in $1 \mathrm{~s} \mathrm{~K}$ shell states individual molecular group atoms, and the detailed spectroscopy of unoccupied near vacuum level states of solvent and solute molecules by resonant Auger spectroscopy, in summary, I feel strongly compelled to thank very many coauthoring colleagues who were with me on this journey at different times over more than three decades. For names I can refer here, only, to the shorter list of coauthors given in the cited references, although it was many more people who have lent their hands, discussed ideas and kept the projects going by their support. Also, I gratefully acknowledge the continuing support by my home institution, Max-Planck-Institut für Strömungsforschung/MPI Dynamics and Self-Organization/, by the Deutsche Forschungsgemeinschaft, by the BESSY synchrotron radiation facility, and by the Max-Born-Institut. 


\section{References}

1. Gasparus Scotus "Technica Curiosa" p. 172, experimentum XXXVIII; Nuremberg 1664 (Digital Edition: Herzog August Library, Wolfenbuittel)

2. M. Faubel, S. Schlemmer, J.P. Toennies, A molecular beam study of the evaporation of water from a liquid jet. Z Phys D Atoms Molecul Clust 10, 269-277 (1988)

3. M. Faubel, Photoelectron spectroscopy at liquid surfaces, Chap. 12, vol. I, in Photoionization and photodetachment, vol. 101A, ed. by C.Y. Ng (World Scientific Publishing, Singapore, 2000), pp. 634-690

4. S. Hess, M. Faubel "Gase und Molekularstrahlen" Fig. 1.70, in: Bergmann-Schäfer Lehrbuch der Experimentalphysik Bd. 5, ed. by K. Kleinermanns, Walter de Gruyter (Berlin, New York 2006), p. 119

5. O. Stern, Nachtrag zu meiner Arbeit: Eine direkte Messung der thermischen Molekularstrahlgeschwindigkeit (Comment to my paper: A direct measurement of the thermal molecular beams velocity). Z. f. Phys. 3, 417-421 (1920) "Zusammenfassung: Es wird die Frage der Geschwindigkeitsverteilung der von einer Flüssigkeitsoberfläche ausgehenden Moleküle diskutiert. Ferner werden die Resultate einiger neuerer Messungen der mittleren Geschwindigkeit von Silberatomen mitgeteilt" 'Summary: I discuss the question of the velocity distribution of molecules emerging from the surface of a liquid. In addition, results of some new measurements of the mean velocity of silver atoms are communicated."

6. M. Faubel, T. Kisters, Non-equilibrium molecular evaporation of carboxylic acid dimers. Nature 339, 527-529 (1989)

7. D. Chandler, Introduction to Modern Statistical Mechanics (Oxford University Press, New York, 1987)

8. M.P. Allen, D.J. Tildesley, Computer Simulation of Liquids (Oxford University Press, Oxford, 1987)

9. J.R. Rumble (ed.), CRC handbook of chemistry and physics, 98th edn. (CRC Press, Taylor \& Francis Ltd, Boca Raton, London, 2017)

10. M. Faubel, B. Steiner, J.P. Toennies, Photoelectron spectroscopy of liquid water, some alcohols, and pure nonane in free micro jets. J. Chem. Phys. 106, 9013 (1997)

11. M. Faubel, B. Winter, Photoemission from liquid aqueous solutions. Chem. Rev. 106(4), 1176$1211(2006)$

12. W.S.M. Werner, W. Smekal, C.J. Powell, NIST database for simulation of electron spectra for surface analysis (U.S. Department of Commerce, National Institute of Standards and Technology, Gaithersburgh MD, 2005)

13. R. Weber, B. Winter, P.M. Schmidt, W. Widdra, I.V. Hertel, M. Dittmar, M. Faubel, Photoemission from aqueous alkali-metal-iodide salt solutions using EUV synchrotron radiation. J. Phys. Chem. B 108(15), 4729-4736 (2004)

14. N. Ottosson, M. Faubel, S.E. Bradforth, P. Jungwirth, B. Winter, Photoelectron spectroscopy of liquid water and aqueous solution: Electron effective attenuation length and emission-angle anisotropy. J. El. Rel. Phen. 177, 60-70 (2010)

15. P. Jungwirth, D.J. Tobias, The molecular structure of salt solutions. J. Phys. Chem. B 105, 10468-10472 (2001)

16. D. Nolting, N. Ottosson, M. Faubel, I.V. Hertel, B. Winter, Pseudoequivalent nitrogen atoms in aqueous imidazole distinguished by chemical shifts in photoelectron spectroscopy. J. Am. Chem. Soc. 130(26), 8150-8151 (2008)

17. T. Lewis, M. Faubel, B. Winter, J.C. Hemminger, $\mathrm{CO} 2$ Capture in amine-based aqueous solution: role of the gas-solution interface. Angew. Chem. Int. Ed. 50, 10178 (2011)

18. B. Winter, M. Faubel, I.V. Hertel, C. Pettenkofer, S.E. Bradforth, B. Jagoda-Cwiklik, L. Cwiklic, P. Jungwirth, Electron binding energies of hydrated $\mathrm{H} 3 \mathrm{O}+$ and $\mathrm{OH}-$ : photoelectron spectroscopy of aqueous acid and base solutions combined with electronic structure calculations. J. Am. Chem. Soc. 128(12), 3864-3865 (2005) 
19. B. Winter, E.F. Aziz, N. Ottosson, M. Faubel, N. Kosugi, I.V. Hertel, Electron dynamics in charge-transfer-to-solvent states of aqueous chloride revealed by $\mathrm{Cl}^{-} 2 \mathrm{p}$ resonant augerelectron spectroscopy. J. Am. Chem. Soc. 130(22), 7130-7138 (2008)

20. E.F. Aziz, N. Ottosson, M. Faubel, I.V. Hertel, B. Winter, Interaction between liquid water and hydroxide revealed by core-hole de-excitation. Nature 455, 89-91 (2008)

Open Access This chapter is licensed under the terms of the Creative Commons Attribution 4.0 International License (http://creativecommons.org/licenses/by/4.0/), which permits use, sharing, adaptation, distribution and reproduction in any medium or format, as long as you give appropriate credit to the original author(s) and the source, provide a link to the Creative Commons license and indicate if changes were made.

The images or other third party material in this chapter are included in the chapter's Creative Commons license, unless indicated otherwise in a credit line to the material. If material is not included in the chapter's Creative Commons license and your intended use is not permitted by statutory regulation or exceeds the permitted use, you will need to obtain permission directly from the copyright holder. 